Service social

\title{
Valeurs, prise de décision et action sociale. Recherche de valeurs : nature et approches. Recension des écrits américains.
}

\section{René Auclair}

Volume 44, numéro 1, 1995

Valeurs, pratiques, action sociale

URI : https://id.erudit.org/iderudit/706679ar

DOI : https://doi.org/10.7202/706679ar

Aller au sommaire du numéro

Éditeur(s)

École de service social de l'Université Laval

ISSN

1708-1734 (numérique)

Découvrir la revue

Citer cet article

Auclair, R. (1995). Valeurs, prise de décision et action sociale. Recherche de valeurs : nature et approches. Recension des écrits américains. Service social, 44(1), 5-48. https://doi.org/10.7202/706679ar
Résumé de l'article

Le présent article a pour objectif de faire connaître les résultats d'une recension des écrits américains portant sur les valeurs, la prise de décision et l'action sociale. Le plan suivi aborde tout à tour la nature des valeurs, les valeurs en tant que composantes de la prise de décision, les conflits de valeurs chez les individus et dans la société, l'enseignement des valeurs à l'école et les objectifs de l'enseignement des valeurs. L'auteur présente finalement une démarche américaine de prise de décision et d'action sociale : a) valeurs, méthode de recherche et approches; b) modèle de recherche des valeurs; $c$ ) analyse et clarification des valeurs; d) stratégies pour l'identification des valeurs; e) prise de décision et stratégies en action sociale. 


\section{A $R$ R $T$ T I C L E E S}

\section{Valeurs, prise de décision et action sociale Recherche des valeurs: nature et approches Recension des écrits américains*}

RENÉ AUCLAIR

Professeur, École de service social

Université Laval

\section{INTRODUCTION}

Le présent article a pour objectif de faire connaître les résultats d'une recension des écrits américains portant sur les valeurs et l'action sociale.

\section{Remarque préliminaire}

Nous aimerions faire $d^{\prime}$ abord la remarque suivante que nous partageons avec des spécialistes des domaines de la philosophie et du service social ${ }^{* *}$.

* Recension tirée de James BANKS et Ambrose A. ClEGG Jr. (1990). Teaching Strategies For the Social Studies: Inquiry, Valuing, and Decision-Making, $4^{\mathrm{e}}$ édition, traduit et présenté par René Auclair. New York: Longman. Chapitre 15: "Valuing, DecisionMaking, and Citizen Action ", p. 427-467.

** Nous remercions MM. Jacques Laforest, professeur titulaire, École de service social, et Louis $\mathrm{O}^{\prime}$ Neill, professeur titulaire, Faculté de théologie, de I'Université Laval qui ont commenté notre article avant sa publication. 
Les auteurs américains postulent au départ qu'on ne peut s'entendre sur la définition des valeurs ni sur leur identification. Ils semblent également faire de la définition des valeurs une opération relevant de la sociologie, alors qu'il s'agit essentiellement d'une perception philosophique. La sociologie décrit les valeurs plus qu'elle ne les définit. On pose également comme postulat qu'il existe une interchangeabilité des valeurs ouvrant la voie à un éventuel remplacement de valeurs par d'autres.

Nous ne préciserons pas davantage à ce moment-ci, puisque nous aurons, en conclusion, l'occasion d'ajouter des commentaires sur cette recension des écrits.

\section{Composante des valeurs de la prise de décision}

Composante essentielle de la prise de décision, la connaissance ne suffit pas. II faut que les intervenants sociaux, dans leurs prises de décision, définissent et clarifient leurs valeurs et les rattachent à la connaissance dont elles dérivent. La composante des valeurs occupe une place importante dans la prise de décision; c'est souvent pour sa valeur que l'on acceptera ou rejettera la connaissance. Une confusion dans la valeur mène souvent à des résultats confus et contradictoires de l'action sociale.

\section{Nature des valeurs}

$\mathrm{Ni}$ la philosophie ni les sciences sociales ne s'entendent vraiment quand il s'agit de définir la valeur. Rokeach la définit ainsi : "type de croyance, au centre du système global des croyances auxquelles on s'attache, qui concerne ce que l'on devrait faire ou ne pas faire, ou qui concerne les fins qui méritent d'être atteintes dans le cours de I'existence» (Rokeach, 1969). Contrairement aux attitudes ou à d'autres croyances, les valeurs ne sont pas reliées à des choses, à des personnes ou à des groupes particuliers, mais elles sont très générales et influent sur le comportement de chacun envers une classe étendue de choses ou de personnes. C'est aussi une mesure que l'on se fixe pour déterminer si quelque chose est bon ou mauvais et pour juger de sa propre conduite. Les gens acquièrent leurs valeurs, comme ils le font pour d'autres croyances et attitudes, en suivant l'exemple des gens qui forment leur environnement social. La plupart ne s'acquièrent pas par la réflexion ou indépendamment les unes des autres. Leur origine sociale explique pourquoi la grande majorité des gens qui partagent la même culture, la même sous-culture ou le même système social sont portés à partager des valeurs semblables. 


\section{Conflits de valeurs chez les individus et dans la société}

La plupart des individus qui composent la société moderne n'acquièrent pas un ensemble bien déterminé de valeurs; ils en assimilent plutôt un grand nombre qui sont contradictoires et qui se heurtent les unes les autres. D'où confusion et conflits de valeurs que l'on retrouve aussi bien chez les individus que dans la société en général. Liberté et égalité composent le credo des Américains, mais dans la pratique ces valeurs entrent souvent en conflit. Les propriétaires qui veulent être libres de refuser la location d'un appartement à des familles non traditionnelles ou à des couples qui ont des enfants ne respectent pas le droit à l'égalité. Shaver et Strong commentent les conflits propres aux valeurs américaines d'égalité, de justice et de liberté : "La liberté de parole empiète sur la tranquillité des citoyens; l'égalité accrue pour les uns - conséquence du mouvement pour les droits de l'homme - a pour résultat de limiter la liberté des autres. On ne connaît pas d'exemple où les valeurs de chacun se soient pleinement réalisées dans la lutte menée par la société pour atteindre l'idéal ultime qu'est la dignité pour tous " (Shaver et Strong, 1976).

Les conflits de valeurs que vivent les jeunes dans nos sociétés modernes et démocratiques sont particulièrement graves, bien davantage qu'ils ne le sont dans les sociétés traditionnelles. Ils ont à choisir entre une grande diversité de valeurs qui sont pour beaucoup d'entre elles contradictoires. Les médias de communication, la folle mobilité de la société moderne mettent sous les yeux de la jeunesse une grande variété de façons de vivre et de croyances. Leurs problèmes sont accentués par le fait qu'on s'attend à ce qu'ils passent rapidement de l'enfance à l'âge adulte. On leur demande, par conséquent, de chevaucher deux ensembles d'idéaux différents I'un de l'autre. Ils sont souvent cyniques quand ils se rendent compte que les adultes ne vivent pas selon l'idéal qu'ils proposent. Ces jeunes voient dans la défaillance des adultes l'absence même d'idéal. L'égalité et l'honnêteté, comme le font remarquer Shaver et Strong, sont des idéaux que peuvent défendre les gens sans toutefois y être fidèles. II est donc souvent difficile pour les jeunes de comprendre et de porter un jugement.

\section{Enseignement des valeurs à l'école}

L'école devrait servir de guide auprès des jeunes pour les aider à identifier et à clarifier leurs valeurs et à en faire un choix intelligent, cela afin de dissiper la confusion qui règne dans les problèmes 
sociaux et individuels au sein de la société moderne. Cependant, beaucoup d'enseignants ne guident pas adéquatement les élèves en ce qui concerne les questions morales (Raths, Harmin, Merrill et Simon, 1978).

Certains enseignants traitent les questions de valeurs comme si elles n'existaient pas. Ils s'appuient sur le fait que si les élèves connaissent les données des problèmes sociaux, ils pourront résoudre ceux-ci. Ces enseignants font montre d'une fausse objectivité. Chez d'autres, c'est l'évasion stratégique. Dès qu'il est question de certaines valeurs dans la classe, ils tâchent de dévier vers un sujet moins problématique. C'est ainsi que des sujets comme le tri génétique, les droits des homosexuels et les scandales politiques sont promptement et volontairement esquivés.

Éduquer aux valeurs jugées bonnes par l'adulte est l'approche probablement la plus fréquente à l'élémentaire et au secondaire. La justice, la vérité, la liberté, l'honnêteté et l'égalité sont enseignées en se référant à des héros légendaires, des histoires, des rituels et des chants patriotiques. Le but principal de la recherche des valeurs dans les écoles américaines devrait être d'aider l'élève à développer son adhésion envers les valeurs auxquelles on croit aux États-Unis: I'égalité et la dignité humaine. Cependant, il ne faut pas endoctriner au détriment d'une saine réflexion qui porterait l'élève à adhérer aux valeurs démocratiques. Une approche rigide viole les valeurs démocratiques et $n^{\prime}$ aide pas l'élève à acquérir une méthode pour trouver et clarifier ses propres valeurs.

L'enseignement qui vise à inculquer les valeurs est aussi discutable parce qu'il laisse supposer que l'élève est incapable de distinguer les valeurs "bonnes ou mauvaises». Mais là n'est pas la question. L'élève peut habituellement distinguer le bien du mal; les problèmes naissent cependant lorsqu'il lui faut choisir entre deux choses jugées bonnes. Par exemple, si un copain lui demande son opinion sur un dessin plus ou moins bien réussi que ce dernier vient d'exécuter, que doit-il faire? Être honnête ou poli envers ce copain? S'il est honnête et dit la vérité, il peinera son copain et, par conséquent, ne sera pas poli. Ce cas illustre comment des valeurs également «bonnes " peuvent devenir contradictoires quand l'enseignant a adopté une méthode d'enseignement dite d'endoctrinement.

Ces méthodes sont également discutables en ce qu'elles ne laissent pas à l'élève la liberté de choisir et ne lui apprennent pas à trouver, clarifier et justifier ses valeurs morales. Alors que nous devrions exiger des élèves qu'ils défendent les valeurs qu'ils ont choisies en tenant compte des valeurs sociales de dignité humaine et 
d'égalité, chaque génération doit avoir la possibilité de définir le sens absolu de ses valeurs. On ne peut s'attendre à ce qu'elles soient le guide de toute une vie, à moins qu'elles aient été choisies librement parmi des solutions de remplacement après un examen approfondi des conséquences de ces solutions. Un sentiment de fierté doit naître des idéaux qui guident la conduite de chaque individu (Raths et al.). $\mathrm{Si}$ les enseignants et les adultes imposent des valeurs, l'élève ne pourra leur accorder de prix et, loin de la présence de ces autorités, elles $\mathrm{n}^{\prime}$ auront que peu d'influence sur sa conduite.

\section{Les objectifs de l'enseignement des valeurs}

L'objectif principal de l'enseignement des valeurs devrait être d'aider l'élève à développer chez lui un ensemble de valeurs qui le guideront vers une action sociale déterminée et réfléchie. La meilleure façon d'atteindre cet objectif est d'utiliser une méthode qui favorisera la découverte des valeurs dans une atmosphère démocratique à I'intérieur de la classe. Dans les classes où l'on s'exerce à la démocratie on offre: la liberté d'expression quant au choix des valeurs; la détermination des conflits qui peuvent surgir entre ces choix; l'examen des solutions de remplacement; la prise en considération des conséquences des différents choix; le choix des valeurs; la défense des choix moraux en tenant compte de la dignité de I'homme ou d'autres valeurs que le peuple américain privilégie. On doit donner à l'élève la possibilité de réfléchir pour trouver ses propres valeurs afin qu'il s'oriente vers le choix de la dignité de l'homme, l'égalité et $d^{\prime}$ autres valeurs démocratiques. On doit encore lui donner la possibilité de réfléchir sur ces valeurs dans une ambiance démocratique pour qu'elles deviennent siennes. Pour en arriver à ce résultat, il faut qu'il puisse exercer un libre choix après avoir pris en considération les conséquences de chacun de ses choix (Raths et al.).

\section{VALEURS, MÉTHODE DE RECHERCHE ET APPROCHES}

Nous avons proposé que l'enseignant devrait aider l'élève à acquérir une méthode pour clarifier et trouver ses propres valeurs plutôt que de les enseigner de façon doctrinale. $C^{\prime}$ est la seule approche qui soit valable pédagogiquement et démocratiquement. Ni les sciences sociales, ni la philosophie n'offrent de modèle standardisé ; plusieurs modèles ont été élaborés qui mettent l'accent sur un choix libre. Parmi ces modèles, citons ceux qu'ont élaborés Hunt et Metcalf 
(1966), Oliver et Shaver (1970), Newmann et Kohlberg (1971). Hunt et Metcalf proposent d'aider l'élève à analyser les questions de valeurs apparentées à ce qu'ils qualifient de "domaines problématiques de la culture». Ce sont: les classes sociales, la religion et la morale, la race et les relations avec les minorités. Grâce à ce modèle, l'élève définira le concept de valeur, prévoira les conséquences, estimera les conséquences $d^{\prime}$ après un ensemble de critères et tentera de justifier les critères d'évaluation des conséquences.

Dans la préparation de leurs principes et de leurs stratégies, Oliver, Shaver et Newmann ont fondé leur hypothèse sur le fait que la plupart des Américains privilégient les valeurs ancrées dans la croyance commune que sont la justice, l'égalité et la dignité humaine. Selon ces auteurs, la controverse publique est d'abord le résultat de valeurs conflictuelles et de diverses interprétations de ces valeurs. Certaines valeurs ont plus d'importance que d'autres. C'est la dignité humaine qui vient en premier lieu chez les Américains. Pour leur part, Shaver et Strong déclarent: «nnous croyons que le point central de la démocratie est la foi en chaque individu, foi en sa valeur et en son mérite en raison de sa qualité d'être humain qui le rend égal à autrui et qui n'est pas fondée sur les richesses matérielles, les dons intellectuels ou les performances physiques. Ce point central se résume en deux mots: dignité humaine» (Shaver et Strong). Newmann s'associe à Oliver et Shaver pour proposer des méthodes qui résoudront la controverse publique par une discussion rationnelle. Les propositions de Newmann sur les points de discussion comportent trois éléments : 1) le problème moral, 2) la définition, 3) l'explication de faits (figure 1).

\section{Figure 1}

\section{Éléments de la controverse classés selon l'approche de Newmann}

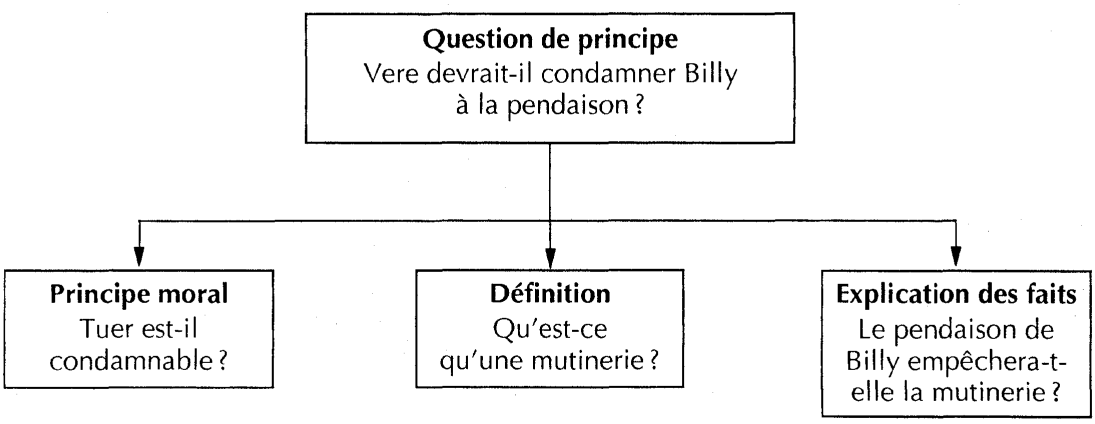

Reproduit avec la permission de Fred N. Newmann (Donald W. Oliver est le co-auteur). Clarifying Public Controversy: An Approach to Teaching Social Studies, Boston: Little, Brown and Company, 1970, p. 43. 
La théorie de Kohlberg sur le développement moral est fortement influencée par la théorie de Piaget sur le développement cognitif. En bref, il établit que la capacité de porter un jugement moral s'opère de façon séquentielle, en une série d'étapes. Il y a, selon Kohlberg, trois niveaux de développement moral: 1) le préconventionnel, 2) le conventionnel, 3) le postconventionnel (Kohlberg et Turiel). Ces niveaux consistent en six étapes qui constituent une hiérarchie et qui s'avancent graduellement vers un raisonnement moral (tableau 1). Dans la première étape, le raisonnement moral est principalement influencé par le désir d'éviter la punition et de respecter l'autorité. Cette étape contraste remarquablement avec la sixième où le raisonnement moral est influencé par des principes universels d'éthique: la justice, l'égalité et l'inviolabilité de la vie humaine.

Kohlberg a déterminé les niveaux de raisonnement moral des individus en analysant leurs réponses à des problèmes concrétisés dans des anecdotes qui présentaient des dilemmes moraux. On demandait à ces personnes d'établir quels actes accomplirait le personnage principal de l'anecdote et quelles étaient les raisons de leur choix. D'après ce même auteur, il y a une relation, mais elle n'est pas directe, entre l'âge chronologique et le développement moral. Le raisonnement des enfants très jeunes se situe dans les premières étapes; plus l'individu vieillit et plus il s'avance vers les dernières étapes. Kohlberg centre sa théorie sur la notion que l'éducation morale doit être basée sur le niveau de développement moral de l'élève et que le raisonnement de ce même élève ne peut atteindre une étape plus élevée que celle où son degré de maturité le situe (Kohlberg et Turiel). Il croit également que si l'école n'offre pas en même temps le degré d'avancement correspondant au développement moral de l'élève, elle n'accroîtra pas son développement moral. Une autre hypothèse est aussi primordiale dans la théorie de Kohlberg: chacun doit avancer pas à pas et ne pas brûler les étapes. Ses recherches lui ont démontré que chez les individus qui participent à des discussions où des problèmes moraux sont examinés, il s'ensuit une croissance morale à condition qu'on leur ait posé des questions dont la teneur est d'une étape plus avancée que leur développement normal. Cette façon de faire aide l'individu à avancer vers une étape supérieure sans I'amener au-delà de cette étape. Le but principal de l'éducation morale, selon le même auteur, serait donc de suivre ce cheminement car il vise à soutenir un progrès constant. 


\section{Tableau 1}

\section{Représentation succincte des six étapes conçues par Kohlberg}

\section{Niveau préconventionnel}

À ce niveau, l'enfant réagit aux habitudes culturelles et aux étiquettes "bien » ou «mal», mais son jugement dépend des conséquences physiques ou hédonistes de ses actes (punitions, récompenses, faveurs), ou encore de l'emprise qu'exerce sur lui la personne qui l'initie aux coutumes de la société qui l'entoure et qui lui apprend à discerner ce qui est bien de ce qui est mauvais. Ce niveau se divise en deux étapes:

- La première étape est orientée vers la punition et l'obéissance. Les conséquences physiques des actions déterminent si elles sont bonnes ou mauvaises sans se soucier de la signification humaine ou de la valeur de ces conséquences. Éviter la punition et respecter aveuglément l'autorité ont leur valeur propre qui n'est pas dictée par un ordre moral découlant de la punition et de l'autorité (cette étape est complétée par la quatrième).

- La deuxième étape est orientée vers l'instrument et le relativisme. La bonne action est celle qui fournit le moyen de satisfaire ses besoins et à l'occasion ceux des autres. Les relations humaines ressemblent à un marché. Justice, réciprocité, partage équitable pour chacun sont là, mais toujours empreints de pragmatisme. La réciprocité s'exprime ainsi : "un petit service en attire un autre». II n'y est pas question de loyauté, gratitude ou justice.

\section{Niveau conventionnel}

À ce niveau, il est primordial de répondre aux attentes de sa famille, de ses pairs ou de sa nation sans égard aux conséquences immédiates et évidentes. Il s'agit non seulement de se conformer à ses opinions personnelles et à l'ordre social, mais d'être loyal, activement loyal, par le maintien, le soutien et la justification de l'ordre, et par l'identification aux personnes ou au groupe engagés dans ce processus. Deux étapes sont inhérentes à ce niveau :

- La troisième étape est orientée vers l'accord interpersonnel. Le bon comportement consiste à plaire à autrui ou à lui venir en aide avec son approbation. On se conforme aux stéréotypes de la majorité. C'est le comportement dit naturel. On juge souvent de la conduite par les intentions qui la motivent. "Il est plein de bonnes intentions » devient important pour la première fois. On cherche l'approbation en étant « aimable et gentil ».

- La quatrième étape est orientée vers la loi et l'ordre. On adopte le principe de l'autorité, des règlements fixes et du maintien de l'ordre social. Le comportement qu'il faut adopter est celui du devoir accompli, du respect de l'autorité et du maintien de l'ordre social pour sa valeur intrinsèque. 


\section{Niveau postconventionnel, autonome ou des principes}

À ce niveau, on observe un net effort pour définir les valeurs morales valides applicables au-delà de l'autorité des groupes ou des personnes qui défendent ces principes et au-delà de l'identification de l'individu avec ces groupes. Ce niveau comporte deux étapes:

- La cinquième étape est orientée vers le contrat social, vers la légalité où les accents utilitaires se manifestent. Les actions conformes à la morale ont tendance à être définies en termes de droits individuels et selon des idéaux qui ont été examinés avec soin et que la société entière approuve. On a parfaitement conscience de la relativité des valeurs et des opinions personnelles tout en mettant l'accent sur les procédures qui permettent d'en arriver à un consensus. À part ce qui est constitutionnellement et démocratiquement admis, le bien est affaire de valeurs "personnelles» et "d'opinion». L'accent est ainsi mis sur "le point de vue légal », mais en soulignant qu'il est possible de changer la loi s'il y a raison suffisante d'utilité sociale (plutôt que de s'en tenir formellement à « la loi et l'ordre » comme à la quatrième étape). En dehors du domaine légal, le libre échange et le contrat social créent ensemble l'obligation. C'est, "officiellement», le principe moral du gouvernement américain et de sa Constitution.

- La sixième étape est orientée vers les principes universels d'éthique. Le droit est défini par décision de la conscience en accord avec des principes d'éthique délibérément choisis qui découlent de la compréhension logique, de l'universalité et de la cohérence. Ces principes sont abstraits et éthiques (la Règle d'or, l'impératif catégorique); ce ne sont pas des règles de morale concrètes comme les dix commandements. Ce sont des principes universels de justice, de réciprocité et d'égalité des droits, et de respect de la dignité de chaque être humain.

Source : Lawrence Kohlberg, "The Claim to Moral Adequacy of a Highest Stage of Moral Judgment ", The Journal of Philosophy, vol. 70, $\mathrm{n}^{\circ}$ 18, p. 631-632, oct. 1973. Réimprimé avec la permission de l'auteur.

\section{MODÈLE DE RECHERCHE DES VALEURS}

L'exposé a porté sur la nature des valeurs. La position défendue sur l'enseignement des valeurs favorise la réflexion et le libre choix. Plusieurs approches ont été proposées en vue d'un enseignement moral. La principale, résumée par Superka et al., est présentée au tableau 2. Il est maintenant temps de présenter un modèle de valeurs qui s'accorde avec la théorie de l'enseignement des sciences sociales. Cette théorie peut servir à enseigner les valeurs ou à donner à l'élève l'occasion d'accroître ses habiletés à prendre des décisions. 
Ce modèle s'accorde avec la position défendue sur l'enseignement des valeurs. II permet à l'élève de reconnaître les sources de ses valeurs et celles des autres, de déterminer comment les valeurs de chacun entrent en conflit, de définir les solutions de remplacement de ces valeurs, de prévoir les conséquences de ces solutions, de choisir librement parmi les valeurs qu'ils peuvent identifier et de justifier leurs choix moraux en fonction des valeurs d'égalité et de dignité humaine, valeurs sûres du credo du peuple américain. Il faut être bien persuadé que l'élève doit choisir librement ses propres valeurs pour que cette stratégie soit réalisée avec succès dans la classe. De même, il faut aider l'élève à découvrir les conséquences des différentes valeurs. Les valeurs qu'il choisit doivent s'accorder les unes avec les autres; il doit être capable de défendre et de justifier ses choix moraux avec comme toile de fond la dignité humaine et, enfin, il doit agir selon ses croyances et en accepter les conséquences.

Lorsqu'on enseigne la recherche des valeurs aux élèves, il faut tenir rigoureusement compte de leur âge et de leur niveau de développement. On ne devrait pas exiger de l'élève qu'il raisonne au-delà de ce que lui permet son développement moral. La plupart des enfants du primaire sont incapables de raisonnements complexes sur des questions qui comportent des principes d'éthique de niveau supérieur. Ils sont toutefois en mesure de comprendre des histoires simples où l'on illustre des valeurs d'honnêteté, de vérité et de loyauté. Les enseignants compréhensifs accorderont soigneusement leur enseignement sur les valeurs au développement moral et intellectuel de leurs élèves.

\section{Définition et reconnaissance des problèmes de valeurs: observation et discrimination}

Une réflexion intelligente sur les valeurs et la résolution de problèmes exige que l'élève soit capable de reconnaître les composantes des valeurs lorsque surgit un problème et qu'il lui faut prendre une décision. Il lui faut aussi discerner les problèmes définitionnels, de même que ceux qui concernent la matière et les valeurs. Les études de cas, les romans et les contes, I'information basée sur les faits et les histoires où il faut imaginer une fin peuvent être utiles pour apprendre à l'élève à reconnaître les valeurs qui se trouvent dans les problèmes à résoudre.

Pour aider l'élève à développer son habileté dans la définition et la reconnaissance des valeurs qui entrent en cause dans les problèmes à résoudre, il y a les histoires où il faut imaginer une fin, par exemple 
"Le revers de la fortune» (F.R. et G. Shaftel). Une fois I'histoire lue, il faut poser des questions comme celles-ci: Quels sont les problèmes qui se présentent dans cette historiette? Qu'est-ce qui est important pour les personnages?

\section{Finders Weepers ou Le revers de fortune}

Cette historiette écrite par les Shaftel met en scène trois garçons, Eddie, Pete et Tom, qui sont en visite chez l'oncle d'Eddie pour une semaine. L'oncle Ross leur souhaite beaucoup de plaisir et leur permet de s'amuser dans le lac, mais il leur recommande de ne pas se servir de sa barque. Pendant que Eddie et Tom se baignent, Pete, oubliant la promesse faite à l'oncle Ross, monte dans la barque et se promène sur le lac. Il est bientôt rejoint par Eddie et Tom et les trois garçons s'amusent ferme avec la barque, non sans que Eddie réprimande Pete. À la fin de la semaine, ils s'aperçoivent que la barque $\mathrm{n}^{\prime}$ est plus à sa place. Ils la retrouvent à un kilomètre plus loin, malheureusement endommagée. II y a pour $30 \$$ de réparations et les trois garçons ne disposent que de 11 \$ hormis l'argent qu'ils gardent pour payer leur retour en autobus à la maison. Soudain, à la gare, ils trouvent un portemonnaie qui contient la somme de $22 \$$ et un chèque de $292 \$$. Pete essaie de convaincre ses camarades de garder l'argent et de déposer le porte-monnaie et le chèque au comptoir des objets perdus. Pour sa part, Eddie veut absolument déposer le tout, c'est-à-dire argent, chèque et portemonnaie. Ils décident de voter et c'est la voix de Tom qui tranchera la question.

\section{Description de comportement approprié aux valeurs : description et discrimination}

L'élève identifie le comportement des personnages qui figurent dans I'histoire, l'événement ou la situation. L'action n'a pas besoin d'être décrite en détail; il suffit de décrire le comportement qui illustre la question que $I^{\prime}$ on veut discuter. S'il s'agit d'une histoire dont on doit imaginer la conclusion, par exemple "Le revers de la fortune», I'élève devrait décrire brièvement le comportement des personnages: I'oncle Ross, Pete, Eddie et Tom. II n'est pas nécessaire de rapporter les paroles de chacun, mais plutôt de résumer leur conduite en une ou deux phrases. II faudrait dire de Pete qu'il a été le premier à se servir de la barque et qu'il a voulu garder l'argent trouvé dans le porte-monnaie avant de déposer celui-ci au comptoir des objets 


\section{TAbleaU 2}

\section{Typologie de I'enseignement des valeurs}

\section{Approches}

Inculcation

Développement

moral

Analyse

Clarification

Apprentissage

de l'action
Objectifs

Inculquer certaines valeurs à l'élève. Changer les valeurs de l'élève pour l'amener à réfléchir sur certaines valeurs désirées.

Aider l'élève à élaborer des modèles de raisonnement fondés sur un ensemble de valeurs supérieures. Pousser l'élève à exposer les raisons de son choix de valeurs et ses positions, non seulement pour partager avec les autres, mais pour stimuler chez lui les étapes de raisonnement.

Aider l'élève à penser avec logique et avec un esprit scientifique pour juger les problèmes et les questions de valeurs. Aider l'élève à se servir de processus analytiques et rationnels pour établir des relations entre les valeurs et pour les conceptualiser.

Aider l'élève à connaître et à identifier ses propres valeurs et celles des autres. Aider l'élève à communiquer ouvertement et honnêtement avec les autres au sujet de leurs valeurs. Aider l'élève à examiner rationnellement ses sentiments personnels, ses valeurs et ses modèles de comportement sans en négliger les aspects émotionnels.

Objectifs classés pour l'analyse et la clarification. Fournir à l'élève les occasions d'action personnelle et sociale basée sur ses façons de voir. Encourager l'élève à se voir lui-même comme un être personnel et social, pas complètement autonome, mais membre d'une communauté ou d'un système social.

\section{Méthodes}

Présentation des modèles, renforcement positif et négatif; moquerie; remarques continuelles; manipulation des solutions de rechange; présentation de données incomplètes ou biaisées; jeux et simulations ; jeux de rôle; apprentissage de la découverte.

Épisodes qui posent des dilemmes moraux où sont réunis des petits groupes relativement structurés et capables d'apporter des arguments et de discuter.

Discussion rationnelle et structurée qui exige du raisonnement autant que de l'évidence * test des principes; analyse des cas semblables; débats; recherche.

leux de rôle; simulations; situations artificielles ou chargées de valeurs réelles; exercices d'analyse de soi activités de sensibilisation de l'étudiant; activités l'extérieur de la classe; discussions en petits groupes.

Méthodes classées pour l'analyse et la clarification et également pour les projets d'action à l'école et dans les communautés dans des groupes organisés et par les relations interpersonnelles.

\section{Titres}

Human Values

Series

Coronado Plan:

Teacher's Guide

First Things Values

Teaching Strategies for Moral Dilemmas

Public Issues Series Analysis of Public Issues Program

Values Education

Decision and Outcomes Values and Teaching Values Clarification

Values in Action

Scholastic Contact Series A Problem into Values

Finding Community

Social Action

\section{Auteurs}

Blanchette et al. (1970)

Bensley (1974)

Kohlberg et Selman (1970)

Galbraith et Jones (1975)

Oliver et Newmann (1967-1972)

Shaver et Larkins (1973) Metcalf (1971)

Gelatt et al. (1973)

Roths et al. (1966)

Simon et al. (1972)

Shaftel and Shaftel (1970)

Goody-Koontz (1968-74)

Church (1973)

Jones (1971)

Newmann (1972)

Source : Douglas P. Superka, Christine Ahrens, Judith E. Hedstrom, Luther J. Ford et Patricia Johnson, Values Education. Sourcebook : Conceptual Approaches, Materials Analysis and an Annotated Bibliography. 
perdus. Des questions peuvent être posées pour aider l'élève à décrire exactement le comportement particulier de chacun: "Que fait Pete?", "Que font Eddie et Tom?". Dans la description d'un comportement, l'élève devrait faire le moins possible de déductions. On devrait lui enseigner à faire clairement la distinction entre observation, déductions et jugements de valeur au cours de cette deuxième phase (Lippitt, Fox et Schaible, 1969).

\section{Désignation des valeurs concrétisées dans le comportement décrit : identification, description et hypothèse}

L'élève essaie de désigner par leurs noms les valeurs mises en évidence par les comportements décrits dans la deuxième phase. Un moyen de faciliter l'exercice est de faire une liste des comportements sur l'un des côtés du tableau et, sur l'autre, la liste des valeurs qui en découlent. Au cours de ce processus, l'élève fera des déductions et ne sera pas nécessairement $d^{\prime}$ accord avec les valeurs qui auront été identifiées dans le comportement décrit. Les idées doivent s'exprimer librement à ce stade-ci du processus ; cependant, I'enseignant devrait exiger de l'élève qu'il donne les raisons qui le font identifier telle ou telle valeur. Il faut également exiger que la description du comportement et des valeurs soit aussi précise que possible même si l'élève n'a qu'une information limitée au sujet des personnages étudiés.

Au cours de la discussion sur "Le revers de la fortune », on peut poser à l'élève les questions suivantes: "Comment la conduite de Pete nous révèle-t-elle ce qui compte le plus pour lui ? Qu'est-ce qui compte le plus pour Pete, être honnête ou se sortir du pétrin? Qu'estce qui compte le plus pour Eddie? Et pour I'oncle Ross? Dans le jeu de rôle où l'on mime cette histoire, qu'est-ce qui importe le plus pour Tom?

\section{Détermination des valeurs en conflit dans le comportement décrit: identification et analyse}

Pour aider l'élève à découvrir qu'il y a dans la société des conflits de valeurs comme il y en a entre les individus, il faut lui demander de donner en exemple des valeurs en conflit chez certains individus et évidentes dans le comportement de différentes personnes. En étudiant dans la classe le cas illustré par "Le revers de la fortune", on peut demander à l'élève de décrire les valeurs en conflit chez Eddie qui voulait bien s'amuser avec la barque de l'oncle Ross tout en ne 
voulant pas indisposer celui-ci à son égard. Pete s'est servi le premier de la barque, mais Eddie et Tom lui ont emboîté le pas et se sont également amusés avec la barque. Eddie voulait à la fois s'amuser et plaire à son oncle. Il y a donc conflit entre ces deux tendances.

On peut aider l'élève des classes moyennes à développer son habileté à identifier les conflits de valeurs en étudiant $d^{\prime} i m p o r t a n t e s$ décisions historiques où sont vécus des conflits de valeurs par d'anciens chefs politiques. Président des États-Unis en 1898, McKinley a vécu des moments angoissants. II devait décider soit d'accorder aux Philippines leur indépendance après la guerre hispano-américaine, soit de les annexer aux États-Unis. Dans un document intéressant, il révèle à un groupe religieux qu'il faisait les cent pas la nuit dans la Maison Blanche en priant. Il se décida finalement pour l'annexion. Par l'étude de cette décision importante, l'élève peut analyser les facteurs politiques, culturels et éthiques qui ont influé sur la décision du président McKinley. Beaucoup d'Américains n'approuvèrent pas la décision du Président, dont Samuel Clemens (Mark Twain). Une discussion pourrait s'élever où l'on débattrait des valeurs en conflit entre le Président et Twain.

\section{Hypothèses sur les sources des valeurs analysées: citation des données sur lesquelles sont fondées les hypothèses}

L'élève émet des hypothèses sur la source des valeurs identifiées à la troisième phase. Comme il convient de faire lorsqu'on énonce une hypothèse, il faut exiger de l'élève qu'il apporte les raisons de son jugement. Une hypothèse doit se fonder sur la raison et l'évidence et non pas sur de vagues conjectures. Cette phase d'identification des valeurs aidera l'élève à découvrir que la plupart des valeurs proviennent des personnes qui forment l'environnement de chacun; elles ne sont ni indépendantes ni attribuables à un travail de réflexion. Une fois établie la source des valeurs qui lui sont propres et de celles d'autrui, l'élève sera en mesure d'en apprécier la justesse et la pertinence lorsqu'elles s'appliquent à diverses situations.

Au cours du débat sur "Le revers de la fortune", l'élève peut énoncer des hypothèses sur le pourquoi des valeurs qui sont propres à I'oncle Ross, Pete et Eddie. II sera aussi en mesure de discuter des valeurs qui étaient propres au président McKinley et à Mark Twain et d'ainsi expliquer la divergence de leurs vues. L'émission d'une hypothèse au sujet des sources des valeurs doit être démontrée par des exemples et par l'évidence. 


\section{Mention des valeurs autres que celles citées dans le comportement observé: rappel}

L'élève devrait savoir que dans notre société il est possible de choisir entre un certain nombre de valeurs et qu'il y a, par conséquent, des solutions de rechange qu'il doit connaître s'il a à trouver ses propres valeurs et $s^{\prime}$ il veut être satisfait de ses choix. On ne peut faire un bon choix s'il n'y a pas de ces valeurs de remplacement qu'il faut amener l'élève à découvrir. En jouant "Le revers de la fortune», il faudra qu'il trouve des valeurs de rechange qui pourraient découler du comportement des personnages: Eddie voulait s'amuser et tenait, plus qu'à toute autre chose, à ne pas avoir d'embêtements. À quoi d'autre aurait-il pu tenir? En étudiant la décision du président McKinley au sujet des Philippines, on peut demander à l'élève: Le Président a-t-il voulu absolument faire partager aux Philippins le style de vie des Américains et maintenir la force militaire dans le Pacifique? Aurait-il pu favoriser d'autres valeurs?

\section{Hypothèses sur les conséquences possibles des valeurs analysées: prévision, comparaison, contraste}

Les objectifs du présent paragraphe sont importants pour l'identification des valeurs: 1) ils amèneront l'élève à se rendre compte que de valeurs différentes résultent des conséquences différentes, 2) ils l'aideront à apprendre qu'il faut accepter les conséquences des valeurs que I'on privilégie et 3 ) ils lui feront prendre conscience des conséquences des différentes croyances. Au cours du débat sur «Le revers de la fortune ", la classe peut discuter de ce qui serait arrivé si Pete, Eddie et Tom avaient gardé I'argent et n'avaient porté que le porte-monnaie et le chèque aux objets perdus. Le propriétaire, au moment de récupérer son porte-monnaie, aurait pu les accuser de vol. S'ils remettent le tout intact, ils devront demander à leurs parents de payer les réparations à la barque et faire de petits travaux pour rendre l'argent emprunté. S'il y a discussion sur l'annexion des Philippines par les États-Unis, il pourra être question des conséquences actuelles de la décision du Président (une de ces conséquences est une révolte contre les États-Unis). C'est alors que l'élève pourra émettre des hypothèses sur ce qui serait arrivé si le président McKinley avait décidé de soutenir les Philippines dans leur désir d'indépendance. 


\section{Déclaration des valeurs préférées : choix}

Après que l'élève a décrit le comportement des personnages rencontrés dans les histoires, les cas et les situations présentés, après l'identification des valeurs et des conflits qui peuvent surgir entre elles, après la prédiction des conséquences possibles, il faudrait demander à l'élève de déclarer quelles sont les valeurs qu'il préfère. C'est une étape extrêmement importante qui devrait être franchie avec délicatesse.

Cette phase de recherche des valeurs doit être menée avec prudence, l'enseignant devant éviter de condamner des valeurs qui ne coïncident pas avec ses croyances. Cela ne signifie pas qu'il doive rester neutre sur les questions de valeurs, mais plutôt qu'il doit éviter de se prononcer sur ses choix personnels tant que l'élève $\mathrm{n}^{\prime}$ a pas exprimé les siens. Sinon, l'élève ne trouvera pas dans la classe I'atmosphère qui encouragera l'expression de ses véritables croyances. L'identification des valeurs deviendra un jeu par lequel l'élève tâchera d'imaginer la réponse que l'enseignant désire de lui. Ce n'est qu'en dehors de la classe et parmi ses amis qu'il fera un récit mimé et qu'il exprimera librement ses véritables pensées. Si l'élève sait que I'enseignant pense que Pete, Eddie et Tom devraient retourner le porte-monnaie intact, la leçon à tirer de «Le revers de la fortune » ne portera pas fruit. Les choix doivent s'exprimer librement dans une atmosphère démocratique au sein de la classe et après mûre réflexion de la part de l'élève sur les conséquences de ses prises de position.

\section{9. Énoncé des raisons, des sources et des conséquences possibles des choix de valeurs : justification, hypothèses et prédiction}

L'enseignant a la responsabilité d'aider l'élève à découvrir ses valeurs dans une atmosphère démocratique, mais il doit, de plus, l'aider à déterminer les sources de ses valeurs, les raisons pour lesquelles il les adopte, leurs conséquences possibles; il doit le guider dans la défense de ses choix moraux en vertu des valeurs américaines d'égalité, de justice et de dignité humaine. Les intervenants sociaux réfléchis ont des valeurs claires, il savent pourquoi ils embrassent telles croyances et connaissent les sources et les conséquences de leurs choix. L'élève qui connaît profondément les sources et les conséquences de ses valeurs va vraisemblablement bien réfléchir avant d'adopter d'autres croyances et agir selon celles qu'il a adoptées. Il est à supposer qu'il endossera les valeurs de justice et de dignité humaine véhiculées dans le credo américain. 
Des questions stratégiques peuvent servir pour aider l'élève à énoncer et défendre ses choix moraux. Les questions suivantes peuvent être posées à différents élèves : "Que devraient faire Pete, Eddie et Tom du porte-monnaie? Pourquoi? Qu'avez-vous considéré en prenant votre décision? Comment votre décision indique-t-elle ce que vous estimez le plus important dans la situation? Agiriez-vous $\mathrm{d}^{\prime}$ après cette décision? Est-ce une bonne décision? Pourquoi oui ou pourquoi non? " II faut être prudent lorsqu'on pose ce genre de questions afin d'éviter qu'elles trompent l'élève ou le punissent d'exprimer librement ses croyances et ses valeurs. L'élève doit en effet pouvoir exprimer en toute liberté ses attitudes, croyances et valeurs pour que l'identification des valeurs ait lieu.

\section{Tableau 3}

\section{Modèle pour la recherche des valeurs}

1. Définition et reconnaissance des problèmes de valeurs : observation et discrimination.

2. Description de comportement approprié aux valeurs; description et discrimination.

3. Désignation des valeurs concrétisées dans le comportement décrit: identification, description et hypothèse.

4. Détermination des conflits de valeurs dans le comportement décrit: identification et analyse.

5. Hypothèses sur les sources des valeurs analysées : citation des données sur lesquelles sont fondées les hypothèses.

6. Nom des valeurs autres que celles citées dans le comportement observé: rappel.

7. Émission d'hypothèses sur les conséquences possibles des valeurs analysées : prédiction, comparaison et contraste.

8. Déclaration des valeurs préférées : choix.

9. Énoncé des raisons, des sources et des conséquences possibles du choix des valeurs : justification, hypothèses et prédiction.

\section{Analyse et clarification des valeurs}

Les points développés dans le modèle de recherche des valeurs sont résumés dans le tableau 3 et dans la figure 2. Ce modèle peut servir de processus et aider l'élève à identifier, clarifier ses valeurs et justifier ses choix moraux. Quoique les divers points soient étudiés 
FIGURE 2

Illustration graphique de l'identification des valeurs

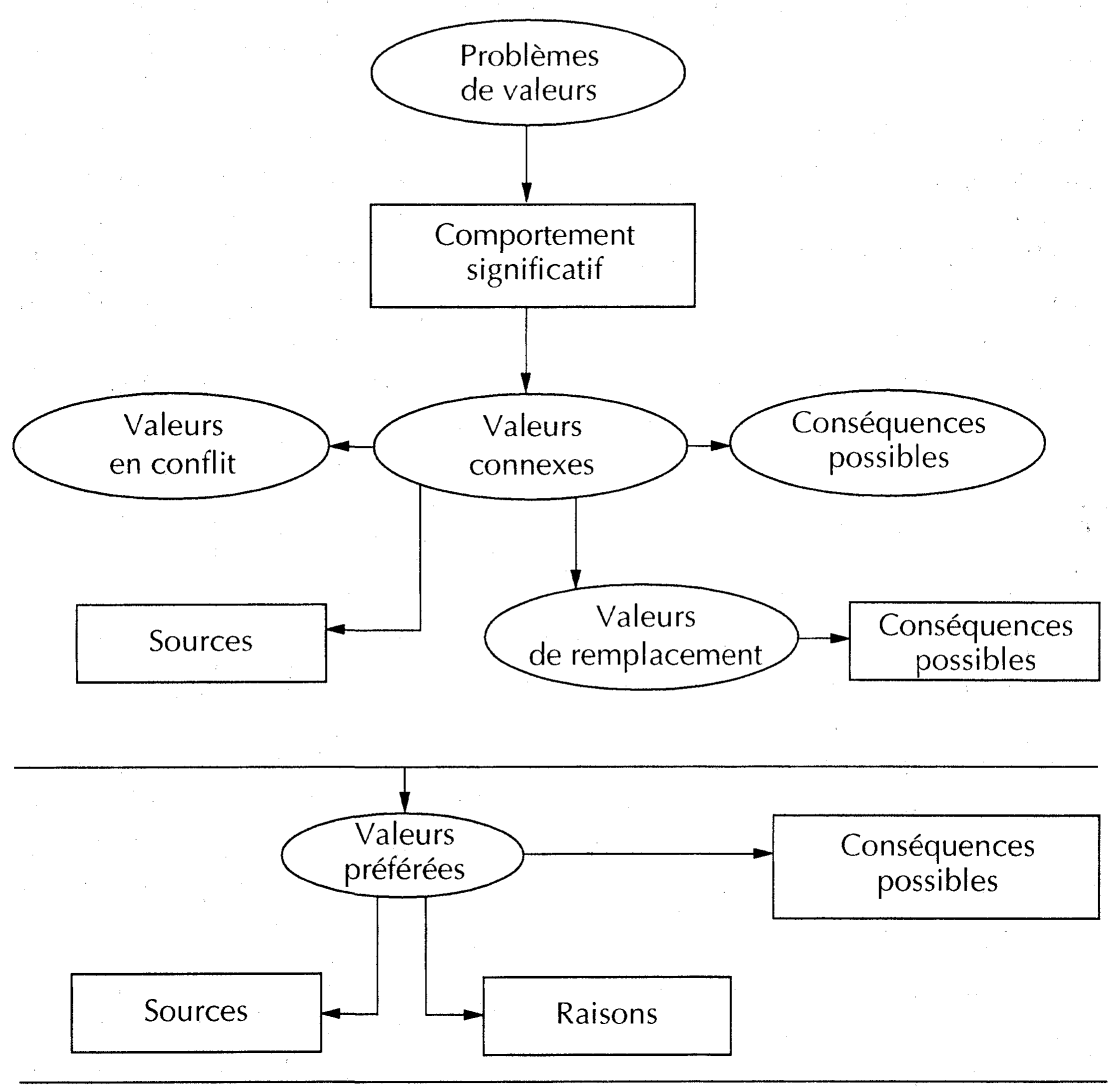

séparément, en pratique ils ne paraîtront pas séparés les uns des autres; ils seront, au contraire, en étroite relation. Par exemple, l'élève peut être amené à décrire un comportement dont les valeurs sont significatives et, en même temps, à nommer les valeurs qui sont ainsi offertes à l'observation. Cependant, si le modèle est partagé en divers points, c'est pour mettre l'accent sur la contribution des valeurs. On devrait donner à l'élève la possibilité de mettre en pratique tous les points; I'enseignant peut au cours d'une leçon souligner un ou plusieurs des points énoncés. Chaque point peut être enseigné séparément ou comme un tout. Pour que l'élève tire tout le profit possible dans l'identification des valeurs, la leçon doit comporter les deux facettes: la vue d'ensemble et la vue particulière. 


\section{STRATÉGIES POUR L'IDENTIFICATION DES VALEURS}

Quantité de matériaux peuvent servir pour que l'élève mette en pratique les points analysés dans le modèle. Comptes rendus d'événements sociaux, événements historiques, incidents en classe et en récréation, littérature, art, photographie, histoires dont il faut imaginer la fin, jeux de rôle, courts métrages et comptes rendus de journaux sont autant de matériaux utilisables. Des stratégies d'identification des valeurs sont présentées ci-après. Groupées selon la classe de l'élève, elles peuvent être utilisées pour l'aider à accroître son habileté à l'identification des valeurs. Ces stratégies sont présentées à titre d'exemples. Il est possible d'en imaginer d'autres également appropriées à l'enseignement de l'analyse des valeurs et de la clarification des habiletés.

\section{Classes primaires}

1. L'élève peut avoir été placé devant une situation où il doit décider ce qu'il doit faire d'un animal errant, un chien ou un chat par exemple. Le sujet est discuté en classe: Jerry promenait son chien quand, soudain, un chiot courut vers lui cherchant des caresses. Le chien de Jerry était très intéressé par le chiot, il le sentait et le léchait. II répugnait à Jerry d'abandonner le chiot, mais il savait que sa mère ne voulait pas d'un autre animal à la maison et pourtant il était évident que ce petit chien était perdu. Que faire à la place de Jerry?

2. Depuis un an, Suzie économise afin de s'acheter un Polaroïd. Elle a mis de côté une partie de sa petite allocation hebdomadaire, mais ce ne sera pas suffisant pour acheter l'appareil convoité. Justement, elle le voudrait bien pour fêter son anniversaire dans deux semaines. Comme elle quitte la cour de récréation au son de la cloche qui annonce la fin de la journée, elle aperçoit par terre un billet de $20 \$$ tout neuf. Elle le ramasse très vite et le cache dans sa main. Que devrait faire Suzie?

3. Joe a vu Kay regarder sur la copie de Carol pendant le test de science, vendredi dernier. Lundi, en annonçant les résultats, M. Hinkle, I'enseignant, a appris aux élèves que Kay avait obtenu la meilleure note et qu'elle était choisie pour représenter la classe à l'expo-science de l'école. Joe devrait-il apprendre à M. Hinkle qu'il a vu Kay tricher, vendredi dernier?

4. Après la lecture à voix haute du conte des "Trois petits cochons", demander aux élèves quelles sont les valeurs du premier petit 
cochon, du deuxième et du troisième, sans oublier le loup. Écrire au tableau ou sur du papier brouillon les valeurs identifiées. Demander aux élèves de dresser une liste des valeurs trouvées pour chaque animal. Ces valeurs sont-elles appropriées au sujet? Oui ou non? Et pourquoi? Demander aux élèves d'imaginer d'autres fins pour la même histoire et de jouer le rôle de chaque animal. Nommer les valeurs trouvées qui seront différentes avec les fins différentes imaginées.

\section{Classes secondaires}

1. On aidera l'élève à reconnaître les valeurs grâce à des photos sans titre. Les photos devront illustrer la position d'une ou de plusieurs personnes à l'égard des valeurs, par exemple un groupe déambulant avec des pancartes ou d'autres marques visibles pour montrer qu'il manifeste pour protester. Certaines manifestations contre la guerre du Vietnam furent violentes. Les photos doivent montrer des manifestations pacifiques et d'autres violentes. L'élève sera appelé à identifier les valeurs véhiculées par les gens qui protestent et il devra également reconnaître celles des gens qui protestent pour des valeurs opposées. Les photos peuvent illustrer des faits actuels ou historiques.

2. On demandera à l'élève de lire les arguments des chefs politiques qui défendent deux positions contraires: augmentation des budgets alloués à la défense du pays ou diminution de ces dépenses au profit des affaires intérieures. On l'aidera à identifier les valeurs privilégiées par ces différents chefs et les conséquences qui découlent de leur choix. Ensuite, on l'aidera à prendre position sur la question controversée des budgets alloués à la défense et à défendre sa position.

3. La coupe du bois est un exemple de dilapidation rapide des richesses naturelles. On demandera à l'élève d'exposer les positions qu'un individu pourrait prendre en ce qui concerne I'usage des arbres et des produits de la forêt. D'une part, on indiquera la quantité totale de bois utilisé pour la construction des maisons, la fabrication des meubles et de la pâte à papier, ainsi que pour l'alimentation des foyers des maisons. D'autre part, on exposera la position des conservateurs qui voudraient que I'on s'abstienne dorénavant de couper du bois, que l'on réensemence les forêts qui ont brûlé et où l'on a trop coupé, et que I'on utilise à la place du bois de nouveaux matériaux de synthèse. 
4. L'étude de I'histoire des États-Unis fournira l'occasion de lire I'acte par lequel Lincoln proclama l'abolition de l'esclavage en 1863. Par cet acte, tous les esclaves étaient libres dans les États qui étaient en révolte contre I'Union. Quels sont les principaux facteurs qui ont influencé Lincoln? Jusqu'à quel point sa décision était-elle politique? ou éthique? On demandera aussi à l'élève de faire l'examen des facteurs moraux et politiques qui ont poussé le président Andrew Jackson à appuyer la loi intitulée Indian Resettlement Act de 1830. Ensuite, l'élève sera appelé à se prononcer sur la décision du président Truman d'autoriser l'usage de la bombe atomique sur le Japon en 1945 et sur celle du président Kennedy d'autoriser un attentat pour renverser le gouvernement cubain en 1961.

\section{Classes supérieures}

1. L'anecdote que voici sera lue en classe. Trois élèves sont rassemblés pour fumer en cachette. Ils s'exercent maladroitement à tenir une cigarette entre leurs lèvres; la fumée qu'ils inhalent les fait tousser tant et plus, mais ils essaient tout de même de se donner des airs de fumeurs expérimentés. De retour chez lui, un des garçons passe, décontracté, devant son père à qui n'échappe pas l'odeur de tabac qui se dégage des vêtements de son fils; il lui demande s'il a fumé. En classe, on demandera aux élèves de mimer la situation et $d^{\prime}$ en imaginer la fin, puis de discuter des valeurs qui se dégagent de ce fait.

2. Au cours des années 80 , la peine capitale est redevenue objet de litige aux États-Unis en raison principalement de l'augmentation du taux de criminalité. Plusieurs États l'ont rétablie et au moins l'un d'entre eux a commandé l'exécution de prisonniers qui avaient été condamnés à mort dans les années précédentes. On demandera à l'élève de discuter les arguments de ceux qui sont pour ou contre la peine de mort et de reconnaître les points de vue moraux, politiques et sociaux qui ressortent dans ce débat. L'élève devra prendre position et défendre son opinion.

3. Les groupes de défense du droit à l'égalité des femmes n'ont pas réussi en 1989 à faire amender la Constitution des États-Unis. L'amendement proposé est toujours très controversé. L'élève devra examiner la question sous toutes ses formes et reconnaître les valeurs qui sont en cause. II devra se prononcer et défendre une position et il décrira les valeurs qu'elle lui inspire. 
4. Des valeurs se font concurrence dans la plupart des cas qui sont soumis devant la Cour Suprême des États-Unis. L'élève sera amené à discuter de cas importants, présents ou passés, des valeurs qui sont ou étaient en conflit, et des valeurs que la Cour a défendues. Il devra également examiner les opinions dissidentes dans chaque cas et identifier les valeurs en cause. Trois différends raciaux devraient être étudiés. Ce sont: 1 ) le cas Plessy v. Ferguson (1896) où la Cour décréta que les moyens de transport "égaux mais séparés » respectaient la Constitution; 2) le cas Brown v. Board of Education (1954) où la Cour décréta que la ségrégation dans les écoles était «en elle-même illégale», renversant ainsi sa propre décision dans le cas Plessy; 3) et enfin, le cas Regents of the University of California v. Bakke (1978) où la Cour maintint l'idée de discrimination positive, mais se déclara contre la stricte observation des quotas raciaux.

\section{PRISE DE DÉCISION ET STRATÉGIES EN ACTION SOCIALE}

\section{Développement ordonné des habiletés de prise de décision}

Par un processus de recherche, l'élève doit être en mesure $d^{\prime}$ associer les connaissances entre elles. Il doit également développer et clarifier ses valeurs pour en arriver à des décisions réfléchies et ensuite donner forme à une politique d'action publique. Nous verrons comment un enseignant ou un conseiller scolaire peut planifier le développement de ces habiletés et de celles qui se rattachent à la prise de décision: synthèse, prévision et proposition du déroulement de l'action.

Pour ce faire, l'enseignant doit tenir compte du programme de sciences sociales enseigné dans son district, des ressources disponibles et du bagage scolaire de l'élève. Il y va aussi des propres champs d'intérêt de l'enseignant. Un grand nombre d'enseignants se retrouveront dans les districts scolaires où seulement des modules de recherche en sciences sociales font partie du programme. D'autres se rendront compte que malgré l'importance des valeurs et des habiletés à prendre des décisions, la recherche en sciences sociales devrait constituer la majeure partie du programme de sciences sociales. Cependant, la recherche des valeurs et les habiletés de prise de décision peuvent être enseignées dans le contexte des modules de recherche en sciences sociales, avec les sciences sociales par ellesmêmes et le contenu historique. Ainsi, au moment où l'on étudiera la Révolution américaine, les questions suivantes pourront être posées. 


\section{Questions sur les valeurs}

1. Comment le comportement des Britanniques et des colons nous montre-t-il ce qui était important pour eux?

2. En quoi les choses importantes pour les Britanniques différaientelles de celles des colons?

3. Y a-t-il autre chose qui aurait pu être important pour les Britanniques et les colons?

\section{Prises de décision}

1. Quelles étaient les solutions de remplacement offertes aux colons?

2. Quelles étaient les conséquences possibles de chacune de ces solutions?

3. Sur quelle logique vous appuyez-vous pour soutenir que les solutions proposées étaient possibles?

Une des approches possibles au développement de la recherche, des valeurs et de la prise de décision est d'enseigner ces habiletés dans le contexte des modules de recherche en sciences sociales. Une autre approche consiste à identifier un certain nombre de questions sociales ou de problèmes vitaux, à faire une liste des concepts de sciences sociales associés à ces questions et à planifier des modules distincts pour enseigner les concepts et l'analyse des problèmes liés à ces concepts.

Les connaissances que l'élève acquiert dans le module de recherche pourront s'appliquer quand il se prononcera sur des questions sociales. Les modules de sciences sociales pourront accroître sa compétence à reconnaître ses valeurs et ses habiletés dans la prise de décision. L'action sociale et la participation à des projets pourront aussi être planifiées et réalisées au cours de l'analyse des problèmes sociaux pour que l'élève puisse agir en accord avec certaines décisions qu'il a prises. Il est possible, par exemple, d'enseigner dans un module des concepts qui aideront l'élève à se prononcer sur des questions sociales liées à la course aux armements nucléaires, aux guerres qui sévissent couramment dans le monde et aux conflits internationaux, aux relations entre races différentes dans la communauté et à la diminution de la couche d'ozone. Ces concepts enseignés dans le module de recherche en sciences sociales rendront l'élève apte à prendre des décisions réfléchies et à s'engager socialement pour aider à la résolution de ces problèmes. 
Une fois planifiés pour l'année les problèmes sociaux à étudier, la relation des concepts aux questions sociales devrait demeurer la principale préoccupation, car cette relation donnera à l'élève la connaissance dont il a besoin pour prendre des décisions et s'engager socialement. Parmi les concepts à approfondir, il y a le pouvoir, le droit international, l'autorité, la pénurie. Ils aideront l'élève à analyser les guerres qui surgissent couramment dans le monde ou les conflits internationaux. Par l'étude des concepts de pouvoir et d'autorité, l'élève sera apte à déterminer qui a l'autorité pour déclarer la guerre et y entraîner le pays et comment les citoyens peuvent exercer leur pouvoir pour influencer la politique en ce qui concerne la guerre. Le concept de droit international aidera l'élève à découvrir comment se font les lois internationales et quelles en sont les conséquences habituelles. Les matériaux et les activités reliés au concept de pénurie éclaireront la façon dont les guerres affectent l'économie d'une nation. L'élève découvrira, par exemple, que les guerres accroissent la prospérité et que, lorsqu'elles se terminent, il en résulte un chômage généralisé.

Nous avons exposé deux façons très importantes de planifier I'enseignement de la recherche, des valeurs et des habiletés de prise de décision. Ainsi ces habiletés peuvent être développées en discutant de questions stratégiques dans le cadre des modules de recherche en sciences sociales. Il existe une autre façon de planifier qui consiste à relever un certain nombre de problèmes sociaux, à structurer des modules conceptuels pour donner aux élèves la connaissance nécessaire à la prise de décisions réfléchies en ce qui les concerne et, enfin, à enseigner les modules de prise de décisions associés aux questions sociales, à différents moments de l'année scolaire.

Quoique ces plans présentent certains avantages et soient même les deux plans réalisables pour beaucoup d'enseignants, il existe un troisième plan qui est, nous le croyons, la façon la plus efficace de structurer l'apprentissage des expériences qui permettront à l'élève d'acquérir de la compétence dans la recherche en sciences sociales, dans la recherche des valeurs et la prise de décision, de même que dans les habiletés en action sociale. L'organisation de ce plan sera étudiée séparément et l'on pourra constater que celui-ci est fortement relié aux deux plans déjà exposés et que les stratégies qu'il comporte peuvent être utilisées également dans les plans discutés précédemment. Chaque enseignant sera en mesure d'adapter les procédés et le modèle de prise de décision à son cas particulier ou bien de les utiliser comme ils sont présentés. Le plan d'organisation décrit dans les lignes qui suivent est le plus efficace des trois présentés. 


\section{IDENTIFICATION DES QUESTIONS ET PROBLÈMES SOCIAUX IMPORTANTS}

Le premier pas à faire dans la planification des modules de prise de décision, en appliquant la méthode très efficace que nous préconisons, est d'identifier les problèmes sociaux qui intéressent l'élève et qui ne sont résolus ni localement ni à plus grande échelle dans la société (S.H. Engle et A.S. Ochoa). Les problèmes devraient être marquants et d'un intérêt durable. Les modules de prise de décision ne devraient pas être structurés pour des questions d'un intérêt limité ou temporaire. II est possible de déterminer les problèmes sociaux généralisés au sein d'une société en analysant soigneusement les nouvelles répandues par les médias pendant une certaine période de temps, en étant généralement au courant des événements qui se passent dans sa communauté et dans la société et en écoutant les conversations des élèves et les commentaires qu'ils font en classe au sujet des événements sociaux et politiques.

On peut aussi se servir de questionnaires et de compositions pour déterminer quels sont les problèmes sociaux les plus importants pour l'élève et la communauté. Bien que les problèmes de la société moderne varient fréquemment selon les périodes, il s'en trouve qui sont d'un intérêt durable. Parmi ceux-ci, il y a l'aliénation politique, la pauvreté, l'inégalité des races et des sexes, la pollution et, au cours des récentes années, la course aux armements nucléaires.

\section{MODULES D'ENSEIGNEMENT DE RÉSOLUTION DE PROBLÈMES}

\section{Définition des problèmes}

L'exemple que voici illustre comment on peut faire l'étude d'un problème social dans la classe et, grâce à un processus de prise de décision, décider de l'action sociale à entreprendre. Riverdale est une ville de taille moyenne où des conflits raciaux se sont développés entre Blancs et Noirs. Au cours de l'été dernier, une famille de race noire s'est installée dans un quartier où les Blancs étaient en majorité. L'événement dégénéra en altercation où plusieurs personnes furent blessées, dont deux policiers blancs. Un groupe de citoyens fit campagne pour qu'une loi prescrive la liberté de choix quant au lieu de résidence, mais il fut très peu soutenu. Les relations interraciales restent tendues à Riverdale. 
En réponse à la loi fédérale, un mouvement d'intégration dans les écoles publiques par le ramassage scolaire où Noirs et Blancs seraient confondus commença, mais un mouvement anti-intégrationniste battit les tentatives d'intégration et convoqua la commission scolaire en session extraordinaire. Les élèves de sixième année de I'école Abraham-Lincoln, une école fréquentée exclusivement par des Blancs en banlieue de Riverdale, posent le problème suivant: "Que pouvons-nous faire pour améliorer les relations interraciales dans notre communauté?"

\section{Recherche en sciences sociales (connaissance associée)}

La connaissance de la matière étant une composante essentielle du processus de prise de décision, l'enseignant identifie les concepts de sciences sociales et les généralisations qui s'y rattachent, afin d'aider l'élève à prendre des décisions réfléchies sur le sujet. L'enseignant fait un choix de concepts : la discrimination empruntée à la sociologie, le conflit à l'histoire, la culture à l'anthropologie, la spécialisation à l'économie et le pouvoir aux sciences politiques. Les généralisations qui découlent des différents concepts s'opèrent, puis les idées secondaires qui découlent de la généralisation et des relations entre Blancs et Noirs aux États-Unis sont formulées. Les lignes qui suivent présentent la généralisation et les idées secondaires d'un des concepts déjà mentionnés.

\section{Concept : discrimination}

Structure de la généralisation : les groupes sont souvent victimes de discrimination en raison de leur âge, de leur sexe, de leur race, de leur religion et de leurs différences culturelles.

\section{Idées secondaires}

1. L'esclavage des Noirs était une forme de discrimination.

2. Les Afro-Américains ont connu la discrimination en matière de droit. Les codes et les lois comme la clause grand-père en sont un exemple.

3. Les Afro-Américains ont connu la discrimination dans l'administration de la justice. Le lynchage généralisé du début des années 1900 en est un exemple. 
4. Les Afro-Américains ont connu la discrimination dans I'accès au vote et au gouvernement.

5. Les Afro-Américains ont connu la discrimination dans l'emploi.

\section{Organisation de la leçon}

L'enseignant devrait identifier les concepts clés qui se rapportent au problème social dont il souhaite la résolution en classe; ensuite, il devrait exposer les structures des généralisations et les idées secondaires qui $s^{\prime} y$ rapportent. II pourra alors formuler les stratégies pédagogiques et préciser de quel matériel il se servira pour la partie sociologique du problème étudié. Les concepts et le matériel pédagogique devraient être présentés de façon logique.

Dans l'exemple présenté ci-dessus, I'enseignant peut souhaiter amorcer le module par le premier concept clé (la discrimination), parce que les idées secondaires qui s'y rapportent traitent des débuts des relations entre Blancs et Noirs en Amérique. Quand la question choisie n'a pas besoin d'être traitée chronologiquement les idées secondaires doivent avoir un certain ordre logique. Un autre enseignant peut vouloir traiter le même sujet en mettant en valeur le concept de pouvoir et discuter du mouvement des droits civiques des années 50 et 60 . Quand il s'agit d'organiser les modules d'analyse de problèmes sociaux, l'enseignant doit prendre en considération les intérêts de l'élève, la disponibilité du matériel, ses propres intérêts, les relations des concepts entre eux, le contenu à étudier et la question sociale à analyser.

Voici comment organiser les modules d'apprentissage qui traitent de problèmes sociaux. II s'agit de diviser une feuille de papier en deux et d'écrire sur un côté les concepts clés et les généralisations et de l'autre les activités qui permettront de développer les idées. Le tableau 4 en fournit un modèle.

\section{Recherche de valeurs}

Une fois que l'élève aura eu la possibilité de trouver les généralisations qui ont rapport aux sciences sociales dans le problème qu'il cherche à résoudre, il sera prêt à recevoir des leçons qui le rendront apte à identifier, analyser et clarifier ses valeurs. La recherche des valeurs peut se faire au moyen de cas relatés dans les journaux et revues, d'histoires dont il inventera la conclusion, de photos, de jeux 


\section{Idées maîtresses}

Concept : discrimination

Structure de la généralisation : les groupes sont souvent objets de discrimination en raison de leur âge, leur sexe, leur race, leur religion et leurs différences culturelles.

Idées secondaires : les Noirs ont connu leur part de discrimination dans l'histoire américaine, que ce soit dans les écoles, l'administration de la justice et dans l'emploi.

\section{Activités}

1. Lire des extraits de North Town et Whose Town? de Lorenz Graham.

2. Regarder un film fixe sur l'esclavage des Noirs et faire une liste de ce qui y est discriminatoire.

3. Trouver des doubles de documents sur les codes des esclaves et la clause grand-père et faire des jeux de rôle pour montrer comment ils ont affecté la vie des Noirs.

4. Faire des statistiques sur le nombre de Noirs qui ont été lynchés au début des années 1900 .

5. Lire des comptes rendus, pour en discuter, de la discrimination que connaissent les Noirs dans l'emploi, les écoles et dans I'administration de la justice d'aujourd'hui.

de rôle ou de dilemmes moraux que l'enseignant et l'élève imagineront de concert. Quelques-uns de ces faits peuvent aussi servir à l'élève pour qu'il détecte et clarifie les valeurs qui sont liées au problème social qu'il étudie.

Quand la classe porte sur un problème à résoudre ou sur une question sociale l'enseignant devrait se servir de stratégies des valeurs directement reliées à la question discutée. Il est entendu que dans le module présenté au tableau 4 , il ne saurait être question de pollution des eaux ou de conflit international à moins que ces problèmes ne regardent directement les relations entre Blancs et Noirs aux ÉtatsUnis ou dans les écoles locales.

Les activités reliées aux valeurs du module offert en exemple au tableau 4 seraient les suivantes:

1. Lecture et discussion d'une sélection de romans de Lorenz Graham: South Town, North Town et Whose Town? Dans cette trilogie, Graham décrit les problèmes, les espoirs et les rêves d'une famille noire qui émigre du Sud vers le Nord à la conquête du rêve américain. Ces romans regorgent de conflits de valeurs et de dilemmes. 
2. Lecture et discussion de la crise morale née pendant la Guerre civile. Les discours tenus par les abolitionnistes et leurs adversaires jettent un éclairage sur la crise morale que la Guerre civile engendra dans la conscience nationale.

3. Lecture et discussion sur les problèmes moraux que soulèvent les lettres écrites par Martin Luther King Jr. (Voir Letter from a Birmingham Jail, 16 avril 1963.) Luther King y réagit aux accusations selon lesquelles il serait un agitateur étranger à Birmingham et il y défend ses tactiques de résistance pacifique.

4. Lecture et discussion des questions morales que souligne le discours de Martin Luther King Jr., I have a Dream, prononcé le 28 août 1963 pendant la marche pour la liberté et les emplois à Washington.

5. D'autres militants noirs, dont Malcolm X et Stockley Carmichael, s'impatientèrent devant les tactiques non violentes de King. Lecture et discussion sur les discours et écrits de King, Malcolm X et Carmichael, démonstration de la similitude et des différences de leurs valeurs.

6. Jeux de rôle et discussion de l'intégration dans une école blanche.

7. Visionnement et discussion sur des images de manifestation pacifique pour les droits civiques au cours des années 60 .

8. Lecture et discussion des conflits de valeurs qui sont nés au moment où les institutions fédérales et les États réalisèrent les plans de discrimination positive pour aider l'élimination de la discrimination dans l'emploi au cours des années 70 et 80 .

\section{Prise de décision et action sociale}

Une fois que l'élève a trouvé les généralisations qui se rapportent aux sciences sociales et qu'il a clarifié ses valeurs en ce qui concerne la question sociale étudiée, l'enseignant devrait lui demander de dresser une liste de tout ce qu'il est possible de faire en ce qui concerne les relations interraciales dans sa communauté. L'élève doit prévoir les conséquences de chaque solution envisagée.

Il est très important que les solutions et conséquences soient réalistes et basées sur la connaissance que l'élève a acquise durant la phase de recherche du module. Elles doivent aussi donner lieu à des énoncés intelligents et ne pas se résumer à des suppositions et des idées chimériques. L'élève doit apporter des données et des raisons 


\title{
Tableau 5
}

\section{Actions à entreprendre et leurs possibles conséquences}

\section{Actions à entreprendre en ce qui concerne les rapports interraciaux dans notre communauté}

\author{
Conséquences possibles
}

1. Éviter les actions de groupe.

2. Agir en groupe pour améliorer les sentiments raciaux en classe et dans l'école.

3. Agir en groupe pour améliorer les rapports sociaux dans la société.

4. Ne pas agir en groupe mais individuellement pour améliorer les rapport interraciaux.
1. L'hostilité peut grandir.

2. Davantage de gens peuvent être blessés dans des émeutes raciales.

3. Les Blancs et les Noirs peuvent en arriver à former des sociétés distinctes.

4. L'intégration peut causer dans notre école un véritable conflit racial.

5. On ne nous critiquera pas si nous adoptons une position conservatrice dans notre école et dans la société en général.

6. Les Noirs se verront peut-être refuser plus de droits dans notre communauté.

1. L'hostilité peut se manifester chez les administrateurs, les autres élèves et les enseignants.

2. En y allant prudemment, nous pouvons améliorer les rapports raciaux dans notre école, mais pas nécessairement dans le reste de la société.

3. Nous pouvons susciter de l'intérêt chez les autres élèves et chez les enseignants en aidant à améliorer les relations raciales dans notre école ou dans la société.

1. L'hostilité peut se manifester dans des groupes communautaires.

2. Les sentiments raciaux peuvent s'allumer dans ces groupes.

3. Si les parents ne coopèrent pas ils peuvent être contre ou ne pas soutenir les efforts des élèves.

4. Des efforts prudents et sages en coopération avec les groupements sociaux et religieux influenceront de façon positive les sentiments raciaux.

1. L'effet ne se fera peut-être pas sentir dans notre communauté, mais chacun se sentira mieux qu'avant puisqu'il agira d'après ses valeurs.

2. Les actions de chaque individu peuvent influencer d'autres individus; ainsi les efforts de chacun serviront à améliorer la situation. 
pour appuyer les solutions et conséquences qu'il formule. II serait, par exemple, chimérique qu'un élève envisage d'aller de porte en porte $s^{\prime}$ entretenir avec ses concitoyens du problème racial afin $d^{\prime} y$ trouver une solution. Cela ne veut pas dire que l'information donnée aux gens ne contribuerait pas à la solution du problème, mais il est évident qu'on ne peut penser à résoudre en classe les problèmes raciaux d'une communauté. On peut cependant agir de façon à améliorer sa propre attitude.

L'élève peut illustrer sous forme de tableau les solutions qu'il envisage et leurs conséquences.

\section{Détermination du déroulement de l'action}

Après avoir trouvé les solutions de rechange, prévu leurs conséquences et identifié la connaissance sous-jacente à ces solutions et conséquences, l'élève devrait retenir les solutions qui s'accordent à son échelle de valeurs. Le processus de prise de décision est illustré à la figure 3 .

L'élève devrait se poser la question suivante et y répondre: comment l'action doit-elle se dérouler pour correspondre aux valeurs qui sont les plus importantes pour moi ? L'élève devrait reconnaître ses valeurs et les classer dans un ordre hiérarchique au cours du module des sciences sociales au moment où l'on traite justement des valeurs. Un groupe d'élèves peut décider de mettre en avant la valeur et la dignité de l'individu par-dessus toute autre chose, et de choisir ensuite l'égalité comme autre valeur très importante. II devrait essayer de définir ses valeurs pour les mettre en application et les associer au déroulement qu'il a choisi. L'élève doit résoudre des problèmes comme celui-ci: I'abstention totale d'agir devant la discrimination imposée aux Noirs dans notre communauté correspond-elle à nos valeurs en ce qui concerne la valeur et la dignité de chaque individu? Refusons-nous la valeur et la dignité de chaque individu quand nous refusons d'accorder à tous des chances égales devant la loi, dans l'emploi et dans le droit à l'instruction? Comment agirons-nous si nous voulons que cela corresponde aux valeurs auxquelles nous tenons?

Un autre groupe d'élèves peut se prononcer à l'égard de la règle de la majorité avant toute autre chose pour en venir à la conclusion que si la majorité blanche approuve l'inégalité dans l'emploi et la séparation dans les quartiers, il $n^{\prime} y$ aurait aucune raison $d^{\prime}$ agir pour être en accord avec les valeurs de ces gens. Ce groupe a analysé les résultats d'une enquête récente sur le comportement racial dans la communauté. 
Figure 3

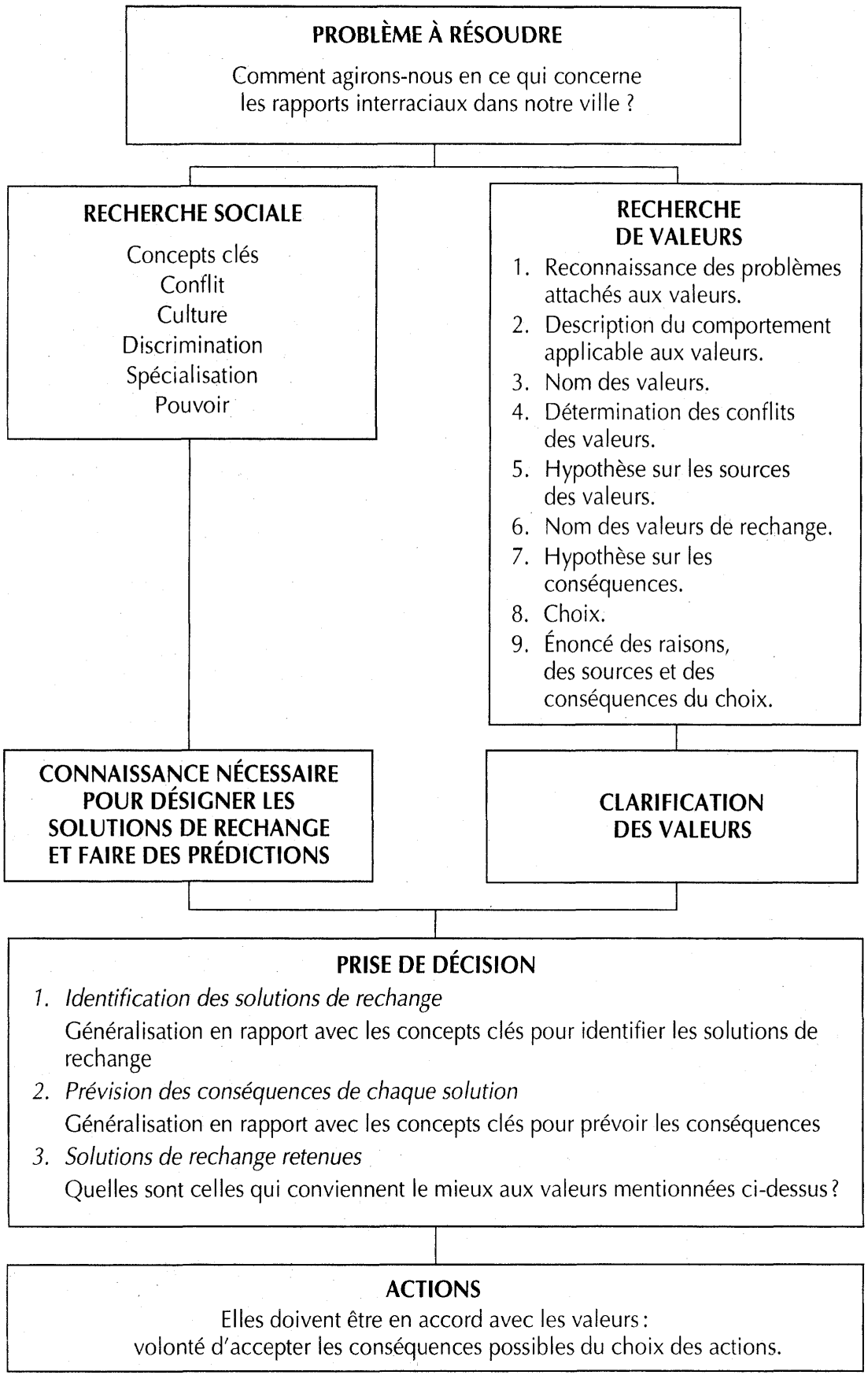


Si un groupe d'élèves en arrive à cette conclusion après mûre réflexion, le rôle de l'enseignant est d'aider ces élèves à réexaminer les sources de leurs valeurs et à déterminer comment elles peuvent entrer en conflit avec d'autres valeurs qu'ils partagent ou avec les valeurs véhiculées dans le credo américain: l'égalité et la dignité humaine. II faudra aussi les aider à reconnaître les conséquences possibles de leurs croyances, déterminer si la vie est possible compte tenu de ces conséquences et s'ils sont fiers de leur choix de valeurs. Pour que se réalise notre théorie en éducation morale, l'élève doit être capable de décision sans intervention coercitive dans une atmosphère démocratique à l'intérieur de la classe. Ce n'est pas facile à réaliser. Cependant, cela correspond à une idéologie démocratique et à un engagement à l'égard du respect de la dignité humaine. Ces valeurs sont celles que nous appuyons et elles constituent le fondement de notre théorie.

On doit encourager l'élève à prendre ses propres décisions, à accepter les responsabilités qui en découlent sans se fier aux autres, y compris les enseignants. On doit I'habituer à élaborer sa propre façon de penser au sujet des questions sociales, mais il se présentera des situations où l'élève devra prendre des décisions en groupe. $C^{\prime}$ est une pratique souhaitable, puisque dans la vie on est souvent appelé à participer à de telles décisions. Cette aptitude est essentielle pour intervenir efficacement dans une société démocratique et pluraliste. $\mathrm{Si}$ des projets d'intervention sociale sont planifiés et réalisés, ils seront habituellement entrepris en groupe, ce qui n'empêche pas certains individus de travailler seuls sur les questions sociales vues en classe.

\section{PRÉVOIR DES OCCASIONS POUR UNE PARTICIPATION À L'ACTION SOCIALE}

Une fois que l'élève a pris des décisions sur des problèmes sociaux, il faudrait, si c'est possible et réalisable, lui fournir des occasions de participer à des projets où se réaliseraient ses décisions. La participation accroît chez l'élève le sens de l'efficacité personnelle, sociale et civique. La connaissance ne vaut pas grand-chose si elle ne sert pas à résoudre les problèmes humains - particulièrement en cette période de notre histoire où les problèmes personnels et sociaux sont au premier plan. Si rien ne se présente pour faire agir l'élève d'après les décisions qu'il a prises (cela ne devrait être ni possible ni désirable), l'école, en coopération avec des organismes publics et privés, devrait 
fournir à l'élève des occasions d'offrir ses services pour mettre en pratique ses décisions. Il est de tradition que les sciences sociales ont plutôt atrophié le sens civique de l'élève plutôt que de l'orienter vers l'efficacité et l'action.

L'action sociale et la participation à des projets peuvent prendre diverses formes: le bénévolat au service de la communauté, des études communautaires comportant une composante d'action, des projets de recherche pour la communauté, des projets internes, une action sociale destinée à influencer la politique sur le plan régional, national et international (Newmann). L'âge et la classe de l'élève sont primordiaux quand il s'agit de planifier l'action sociale et la participation à des projets. Les projets internes réalisés dans les organismes privés et publics ne conviennent généralement pas à des élèves de niveaux primaire et secondaire. On voit cependant un certain nombre de projets internes réussir dans les collèges. L'élève du primaire et du secondaire s'engage souvent dans le bénévolat communautaire et dans les projets de recherche.

On ne s'attend pas à ce que l'élève de ces niveaux résolve des problèmes de société qui résistent à l'action de l'adulte. Il existe tout de même certaines actions qui lui sont propices et qui sont applicables aux grands problèmes sociaux. L'élève contribuera ainsi à sa mesure à l'amélioration de sa classe, de son école et de sa communauté. Et, ce qui est plus important encore, ces actions accroîtront son efficacité personnelle, sociale et civique. Assister l'élève dans le développement de son efficacité personnelle, sociale et civique et de ses habiletés à influencer son environnement social et civique devrait être le but principal de l'action sociale et de la participation à des projets et des activités. Les services à sa communauté sont à considérer, mais ils sont secondaires.

\section{ACTION SOCIALE DANS LA COMMUNAUTÉ ET À l'ÉCOLE}

Dans le cas d'un problème social qui divise toute une communauté les incidents interraciaux que nous avons relatés en sont un exemple -, il vaut probablement mieux que l'élève réserve son action pour la classe, l'école ou son milieu (club ou église) où il ne court pas publiquement le risque qu'on abuse de lui ou qu'on le mette de côté. Si un groupe d'élèves est mêlé de près à une controverse raciale généralisée dans la communauté, l'élève et l'école risquent de devenir la cible de groupes extrémistes. Par décision personnelle, l'élève peut $\mathrm{s}^{\prime}$ engager dans une controverse de ce genre, indépendamment de sa 
classe ou de son école. Dans pareil cas, il devra agir en son propre nom et son action n'engagera pas son école. L'école publique, en raison de sa vulnérabilité dans notre société, sera sévèrement critiquée si les enseignants et les administrateurs ne font pas preuve de bon jugement dans la planification de l'action et de la participation des élèves à des projets.

L'itinéraire que l'élève devra suivre, s'il veut entreprendre une action et prendre exemple sur le problème que nous avons exposé, peut être d'établir un programme de rapports interraciaux ou des leçons d'études ethniques dans sa propre école. Il pourra tout d'abord travailler conjointement avec le principal de l'école, les enseignants, les parents et d'autres personnes de l'école ou du voisinage. L'association du principal d'école, des enseignants, des parents et d'autres personnes de l'école ou du voisinage (APEPP) peut offrir assistance et soutien. Si dans la classe on décide d'entreprendre telle ou telle action, l'élève ne devra jamais être forcé $d^{\prime} y$ participer contre son gré, si cette action est contraire à ses valeurs et à ses croyances.

L'école étant une institution sociale dont les problèmes reflètent ceux de la société, l'élève peut donner forme à une politique publique en travaillant à l'élimination des problèmes de sa classe, de son école ou de son district scolaire. L'étude et l'analyse des problèmes peuvent commencer à l'intérieur de sa classe. Le principal de l'école et I'enseignant doivent être fermement convaincus que l'élève doit participer à la préparation de lignes d'action à l'école et dans la société, ce qui lui fera prendre conscience de sa compétence personnelle, sociale et civique.

Il tient à la volonté de ces deux autorités que les projets d'action de I'élève débouchent sur quelque chose et ce sera en confiant à l'élève la préparation de lignes d'action dans la classe et à l'école. Si l'élève manque d'efficacité personnelle, sociale et civique dans sa propre école, il ne saura tracer de ligne d'action à l'échelle publique ni contribuer à la solution de problèmes sociaux. Tout $n^{\prime}$ est certes pas à la portée de l'élève en ce qui a trait aux politiques scolaires; on ne peut et on ne doit pas lui permettre de tout faire ou de tout influencer. Les administrateurs scolaires et les enseignants ont à se conformer à des règlements et à des lois qui sont décidés en haut lieu. Certains problèmes et des questions qui se font jour à l'école et qui sont de son ressort aideront l'élève à s'affirmer s'il s'engage à les résoudre. Cela peut concerner la discipline, les convenances dans la tenue vestimentaire, les assemblées scolaires, le choix du menu à la cafétéria, les disputes à la récréation, le sexisme et le racisme. La nature et l'importance de la participation de l'élève devraient être 
déterminées par l'ampleur de la question ou du problème et également par la maturité, la connaissance de l'élève et par l'intérêt qu'il porte à ce problème.

L'enseignant désireux de stimuler l'aptitude de l'élève à s'engager socialement doit lui fournir les occasions propices où il pourra s'exercer à clarifier les buts de son action et à mettre en pratique avec succès les habiletés qu'il aura acquises. Un modèle est présenté à la figure 4 où Newmann identifie plusieurs composantes essentielles $d^{\prime}$ une action sociale efficace. Trois points sont ainsi soulignés: a) formulation des buts, b) recherche d'appui aux buts visés, c) résolution des préoccupations psychologiques et philosophiques. L'élève qui formule ses buts en suivant ce modèle articule et justifie ses choix moraux et effectue la recherche de la politique qui s'y rapporte. II explore et expérimente les moyens d'exercer son pouvoir civique quand il cherche les appuis qui maintiendront les buts qu'il s'est fixés. L'engagement social peut être la cause de dilemmes psychologiques et philosophiques pour tout individu. Par conséquent, il est nécessaire de préparer un programme d'action sociale pour aider l'élève à résoudre les préoccupations psychologiques et philosophiques.

FIGURE 4

Champ de compétences requises pour exercer une influence sociale

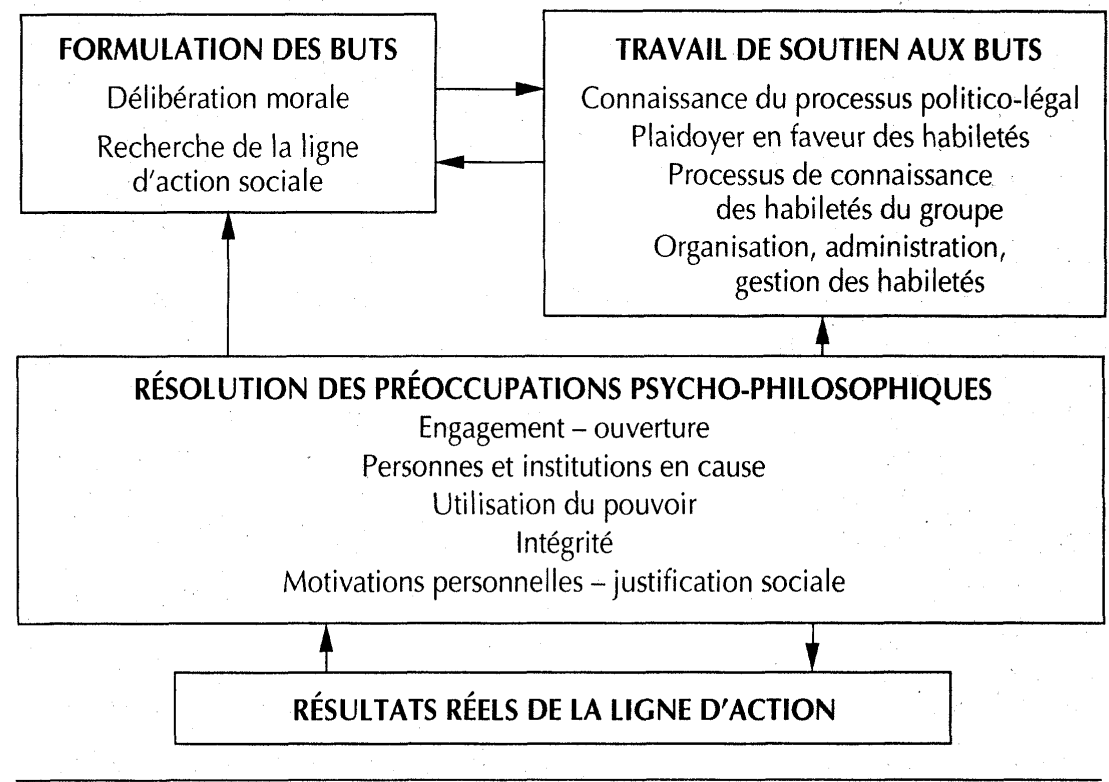




\section{Les lignes directrices pour l'action sociale et la participation sont présentées au tableau 6 . II y a gradation dans les énoncés.}

\section{TABLEAU 6}

\section{Lignes directrices des projets en action sociale}

1. Les activités doivent constituer des expériences significatives et ne pas être que des projets où l'on $s^{\prime}$ engage pour dire que l'on participe dans des projets en action sociale.

2. Le but principal des projets en action sociale n'est pas que de servir la communauté, mais de fournir à l'élève des expériences aux moyens desquelles il pourra acquérir le sens de l'efficacité politique. Cependant, les projets les plus efficaces contribuent à atteindre ces deux buts.

3. Exercer la charité ou autres sortes d'assistance communautaire est légitime et potentiellement significatif, mais, aussi souvent que possible, les projets devraient aider l'élève à acquérir le sens de l'efficacité sociale.

4. Avant de s'engager dans des activités d'action sociale, l'élève devrait avoir étudié les questions qui $s^{\prime} y$ rapportent dans la perspective des sciences sociales, analysé et clarifié ses valeurs en ce qui concerne ces questions, déterminé les conséquences possibles de ses actions et exprimé la volonté d'accepter ces conséquences.

5. Si les problèmes inhérents à l'école peuvent être résolus par l'action de l'élève, la participation aux activités scolaires devrait être prioritaire avant la participation aux projets de la société en général.

6. Si une prise de décision de groupe est légitime et souvent souhaitable, aucun élève n'est obligé d'y prendre part si elle ne correspond pas à ses valeurs et croyances.

7. On devrait prendre en considération l'expérience et l'âge de l'élève au moment de la planification et de la réalisation d'un projet. Un enfant jeune doit se réserver pour sa classe, son école et sa famille ou pour d'autres groupes de niveaux primaire ou secondaire où il se sent en sécurité et où I'on soutient ses actions.

8. La planification des projets en action sociale devrait recevoir le soutien des autres enseignants, élèves, administrateurs scolaires, des organismes communautaires et des membres de la communauté.

9. L'élève qui désire faire de la participation en action sociale devrait bénéficier d'un horaire spécial. Le concept de l'école devrait être élargi ; les activités n'ont pas nécessairement lieu entre les quatre murs de la classe.

10. Si une question sociale divise profondément une communauté, si les émotions sont vécues intensément, les projets devraient être réservés à la classe, à l'école ou à la famille ou à d'autres institutions où l'élève se sent en sécurité.

11. Les actions sociales planifiées par l'élève ne devraient pas contrevenir aux lois et mœurs de la communauté.

12. Les projets planifiés à l'école doivent être en accord avec le credo américain et la dignité humaine.

13. Au moment de la planification des projets à l'école, l'enseignant devrait s'efforcer d'aider l'élève à identifier toutes les conséquences possibles de ses actions, surtout si ces projets peuvent avoir des conséquences indésirables pour l'individu ou le groupe.

14. On ne devrait pas décourager l'élève qui désire s'engager dans un projet individuel, mais on devrait l'amener à réaliser que l'action de groupe est généralement plus efficace que l'action solitaire.

15. Au moment de la planification des projets, l'enseignant doit déployer tous les efforts pour minimiser les préjudices physiques, émotifs ou psychologiques qui peuvent être subis par l'élève. Il sera aidé en cela en sollicitant la coopération de collègues de travail ou ailleurs dans la société et en considérant avec sérieux les conséquences possibles des différents déroulements de l'action.

16. L'action sociale qui s'inscrit dans le programme en sciences sociales ne devrait pas être partisane, Qu'un groupe d'élèves décide de faire campagne pour un candidat ou se prononce sur une question ne devrait pas empêcher que d'autres élèves puissent opter pour des projets parallèles où $s^{\prime}$ exprimeront leur croyances et choix politique. 


\section{PROJETS EN ACTION SOCIALE}

\section{Classes élémentaires}

1. Le module qui abordera la vie de famille donnera I'occasion à l'élève de s'entretenir avec ses parents et grands-parents sur la vie telle qu'elle se passait quand ils étaient jeunes. En collaboration avec l'enseignant, l'élève mettra au point une série de questions que chacun pourra poser à ses parents et grandsparents. Les grands-parents dont I'éloignement ne rend pas faciles les rencontres seront joints par téléphone.

2. L'élève a appris que la pollution était un problème d'importance primordiale. De concert avec d'autres élèves, il a décidé de lutter contre ce problème. Ensemble ils ont mis de côté toutes les boîtes d'aluminium dont on s'est servi à la maison et demandé à leurs voisins de bien vouloir leur donner celles dont il pouvaient disposer. Ils ont vendu leur cueillette au ministère de I'Environnement à raison de 17 cents la livre et ils ont utilisé cet argent pour acheter des plantes et des arbustes pour la cour d'école.

3. Lors d'un module sur la communauté vu en deuxième année, les élèves ont eu à s'occuper de personnes âgées. Ils ont montré beaucoup d'intérêt et ont posé des questions : "Que pouvonsnous faire pour aider les personnes âgées de notre communauté ? » Ils ont décidé de rendre visite à une personne âgée au cours de leurs vacances; ils lui ont apporté des cadeaux et chanté des chansons.

4. Les élèves de troisième année ont appris que, dans certains quartiers de leur propre ville, des gens manquaient de nourriture. Une banque de nourriture créée pour leur venir en aide sollicite des dons. Pendant plusieurs mois, chaque élève a économisé une partie de son allocation pour constituer une somme qui servira à acheter de la nourriture. Ils ont aussi choisi les aliments qu'ils ont offerts.

5. Une classe étudie la vie de famille en Colombie et se met à réfléchir sur la pauvreté vécue dans les autres pays. D'un commun accord, les élèves décident d'envoyer un don à un organisme $d^{\prime}$ aide aux familles de l'Amérique latine. Après s'être renseignés auprès de différentes organisations, ils en choisissent une qui semble le mieux convenir à leurs attentes. Ils ont recueilli l'argent en organisant un "march-o-thon " à l'école et demandé aux voisins de donner 20 cents du kilomètre. 


\section{Classes secondaires}

1. Par l'étude de l'histoire des États-Unis, une classe a appris le rôle de ce pays dans le monde et s'est intéressée a la course aux armements nucléaires. Les élèves de cette classe ont mené une enquête dans l'école à savoir si les élèves étaient au courant de I'escalade nucléaire et si cela les préoccupait. Ils ont ensuite organisé quelques réunions pour exposer les deux facettes du débat sur le nucléaire et le résultat de leur enquête.

2. Au cours d'un module sur l'économie, les élèves ont fait remarquer qu'à chaque rentrée de septembre ils ont du mal à trouver exactement le matériel scolaire que demandent les enseignants. Avec l'accord de leur enseignant, ils ont établi et géré un magasin de fournitures scolaires dans un local inoccupé de leur école. Cela leur a permis de se rendre compte de ce que sont les prix de gros, de détail, le client satisfait et ainsi de suite. Ils ont aussi créé un service d'assistance pour leur école.

3. Grâce à l'argent recueilli en procédant à des lavages de voitures, une classe a pu se rendre au siège du gouvernement de son État afin d'observer le travail des élus. Les élèves ont pu s'entretenir avec certains d'entre eux et ont préparé un compte rendu écrit qui sera soumis à toute l'école.

4. Désireuse d'acquérir un nouveau logiciel de sciences sociales pour l'utilisation d'ordinateurs à l'école, une classe collecte des fonds en vendant des épices aux gens de sa communauté.

5. En s'entretenant avec des personnes âgées et en visitant les musées, cimetières et autres institutions, une classe réussit à faire l'histoire détaillée de sa communauté. Elle en fait le récit sous forme de narration et présente le résultat aux autres classes.

\section{Classes supérieures}

1. Les difficultés budgétaires du district scolaire ont poussé les élèves à entreprendre une campagne d'information dans le voisinage. Ils ont distribué des dépliants et ont demandé aux gens de voter en faveur d'un impôt spécial qui sera alloué au redressement du budget.

2. Un module consacré au rôle de la femme dans la vie américaine a poussé les élèves d'une classe à rencontrer le principal et les enseignants pour discuter de quelle façon on pourrait introduire plus de cours de ce genre dans le programme scolaire. 
3. Un groupe d'élèves a formé un club de travaux dirigés. Les membres de l'équipe ont décidé du jour et de l'heure, en dehors de I'horaire régulier, où ils seraient en mesure de recevoir les élèves ayant besoin de conseils.

4. Après avoir étudié la Déclaration des droits de I'homme et la Constitution, les élèves d'une classe ont mis sur pied un certain nombre d'activités pour faire connaître les droits des élèves. Ils ont organisé une assemblée, un débat et ont invité un conférencier.

5. Au conseil municipal, on a proposé d'appeler une rue du nom de Martin Luther King Jr. Mise au courant de la proposition, une classe a décidé d'assister à une réunion du conseil pour apporter son appui à la proposition.

\section{Résumé}

La connaissance de la matière, la clarification et l'analyse des valeurs faites avec réflexion sont nécessaires aux intervenants sociaux pour qu'ils prennent des décisions nécessaires à la résolution de problèmes personnels, sociaux et civiques, la confusion et les conflits de valeurs marquant les sociétés modernes; pour ces raisons, l'élève a besoin de clarifier et de développer les valeurs d'égalité et de dignité humaine qui correspondent au credo américain. L'élève a besoin qu'on lui enseigne le processus à suivre pour trouver et clarifier ses valeurs. Nous ne pouvons pas nous attendre à ce qu'il affirme des valeurs et y modèle ses actions s'il n'a pas choisi démocratiquement ces valeurs en classe après examen de toutes les solutions de rechange. La première partie de cet exposé est consacrée à revoir des modèles qui peuvent servir à enseigner à l'élève comment trouver et clarifier ses valeurs. Une théorie sur l'enseignement des valeurs et des stratégies de réalisation sont aussi présentées.

On convient que les valeurs sont essentielles au processus de prise de décision. Cela n'empêche pas les intervenants sociaux $d^{\prime}$ avoir à en faire la synthèse avec la connaissance pour décider du déroulement de l'action qui convienne à leur choix de valeurs. La deuxième partie présente un exposé raisonné de prise de décision et $d^{\prime}$ action sociale, un module de prise de décision et, enfin, des exemples d'action sociale et d'activités de participation. Le principal but de l'action sociale et de la participation devrait être d'aider l'élève à développer son efficacité personnelle, sociale et civique. Bien des décisions prises dans les cours de sciences sociales ne pourront être 
mises à exécution par l'élève. Cependant, c'est par la coopération avec des organismes publics et privés que l'élève trouvera des occasions en or de réaliser quelques-unes de ses décisions. L'apport de l'élève à l'amélioration de la vie commune et à la résolution de problèmes sociaux n'est pas de grande importance. Ce qui est bénéfique pour lui c'est qu'il peut acquérir, par ses actions et activités sociales, les attitudes et habiletés qui influenceront son univers personnel, social et civique.

\section{Exercices et questions}

1. Repérer dans une publication récente le cas d'un individu ou $d^{\prime}$ un groupe qui fait face à un problème dont les composantes mettent des valeurs en cause. II peut s'agir d'un couple de Blancs qui se demandent s'ils accepteront que I'on conduise leurs enfants dans une école intégrationniste; $d^{\prime}$ 'une jeune fille ou d'un jeune garçon tenté de faire l'expérience de la drogue, $d^{\prime}$ un organisme qui s'interroge sur le soutien ou le désaccord $q u^{\prime} i l$ devrait donner à un projet d'autoroute indispensable, mais qui nécessite le déménagement des résidents d'un quartier pauvre. Grâce au modèle présenté dans cet article, on peut faire une leçon de valeurs en utilisant un des cas mentionnés ci-dessus.

2. Les images sans titre sont $d^{\prime}$ excellents outils pour enseigner la recherche des valeurs. Repérer dans une revue d'actualité un certain nombre d'images qui illustrent des valeurs, les coller sur un carton et les accompagner d'une série de questions (basées sur le modèle de recherche des valeurs présenté dans cet exposé) que vous pouvez poser à l'élève pour développer ses habiletés dans la recherche des valeurs.

3. Les données descriptives en sciences sociales peuvent évoquer des questions de valeurs. Des articles et des comptes rendus sur la Révolution américaine, l'esclavage, la Guerre civile, la guerre du Vietnam et les problèmes de la société contemporaine offrent beaucoup de possibilités pour la recherche des valeurs. Faire une sélection dans un manuel de sciences sociales et illustrer, en suivant un plan pédagogique, comment la sélection peut être utilisée pour aider l'élève à accroître ses habiletés de recherche des valeurs.

4. À la bibliothèque scolaire, faire l'examen de plusieurs guides et manuels d'enseignement des sciences sociales et évaluer leurs composantes. Le matériel inclut-il les objectifs de recherche des valeurs? Les stratégies de développement des stratégies 
sont-elles fondées? De quelle façon faudrait-il modifier les composantes des valeurs de ce matériel pour les rendre plus efficaces?

5. Quel rôle les valeurs jouent-elles dans la prise de décision? Qu'est-ce que ce rôle implique dans l'enseignement des habiletés de prise de décision?

6. Quel module de rechange peut aider l'élève à acquérir de la compétence dans la prise de décision? Quel plan est selon vous le plus efficace? Pourquoi? Et le plus pratique? Pourquoi?

7. Identifiez un problème social d'actualité et structurez un module de prise de décision pour les élèves du secondaire. Joignez-y les concepts clés et les généralisations qui se rapportent à ce problème et qui concernent les disciplines suivantes: histoire, sociologie, anthropologie, géographie, sciences politiques, économie et psychologie. Planifiez une stratégie pour enseigner la recherche des valeurs. Incluez-y également les stratégies pour enseigner à l'élève comment établir un rapport entre les idées maîtresses et ses valeurs dans le but de prendre des décisions. Dressez la liste des façons dont l'élève peut agir en rapport avec le problème et leurs possibles conséquences.

8. Démontrez la compréhension que vous avez des différents termes que voici en définissant chacun d'eux par écrit. Dites aussi pourquoi chacun est important.
a) valeurs
b) recherche des valeurs
c) conflit de valeurs
d) clarification des valeurs
e) stratégies pédagogiques
f) approche cognitive et progressive de Kohlberg relative à l'éducation morale
g) prise de décision
h) problème à résoudre
i) problème social persistant
j) efficacité personnelle
k) efficacité sociale
l) efficacité civique
m) modèle pour la recherche des valeurs 


\section{CONCLUSION}

Le présent article avait pour objectif de faire connaître les résultats $d^{\prime}$ une recension des écrits américains portant sur les valeurs, la prise de décision et l'action sociale.

Le plan que nous avons suivi aborde tour à tour les valeurs en tant que composantes de la prise de décision, la nature des valeurs, les conflits de valeurs chez les individus et dans la société, I'enseignement des valeurs à l'école et les objectifs de l'enseignement des valeurs.

Nous avons par la suite présenté une démarche américaine de prise de décision et d'action sociale:

- valeurs, méthode de recherche et approches;

- modèle de recherche des valeurs;

- analyse et clarification des valeurs;

- stratégies pour l'identification des valeurs;

- prise de décision et stratégies en action sociale.

La démarche s'avère fort intéressante dans sa proposition $d^{\prime} u n$ contenu et de techniques pédagogiques. II faut néanmoins constater que la stratégie décrite ici présuppose un consensus sur un ensemble de valeurs dont on veut faire la promotion: honnêteté, civisme, justice, non-violence, respect de l'environnement, droit des femmes, etc. Il y aurait donc des valeurs qui s'imposent, dont la reconnaissance dépasse le niveau de la seule subjectivité et qui puissent être transmises par proposition implicite ou explicite. D'où notre préférence pour une définition des valeurs justifiée rationnellement qui s'inscrit à l'intérieur d'une théorie morale qui satisfait aux deux exigences suivantes:

- I'identification d'une valeur fondamentale;

- la portée universelle de cette valeur.

Les auteurs américains dont nous avons recensé les écrits expriment des opinions auxquelles nous ne souscrivons que partiellement lorsqu'ils parlent de la manière de discerner les valeurs, de les proposer et de les transmettre. II est possible que ces divergences résultent en partie d'approches disciplinaires et de sources culturelles différentes. Il est certain par ailleurs que la liberté d'expression est un droit inaliénable. Sans donc nous prononcer formellement sur la validité des énoncés et de la thèse, nous avons estimé qu'il était pertinent, voire utile $d^{\prime}$ en favoriser la communication publique, ne serait-ce que pour provoquer une réflexion sur les valeurs, la prise de décision et I'action sociale au Québec. 


\section{Références bibliographiques}

ENGLE, Shirley H. et Anna S. OCHOA (1988). Education For Democratic Citizenship. Decision Making in the Social Studies. New York: Teachers College Press.

HUNT, Maurice et Lawrence E. METCALF (1968). Teaching High School Social Studies, $2^{\mathrm{e}}$ édition. New York: Harper and Row.

KOHLBERG, Lawrence et Elliot TURIEL (1971). Moral Development and Moral Education, dans Gérald S. Lesser (dir.), Psychology and Educational Practice. Glenview, II. : Scott, Foresman.

LIPPITT, RONAld, Robert Fox et Lucille SCHAIBLE (1969). Social Science Laboratory Units : Teachers Guide. Chicago : Science Research Associates Inc.

NEWMANN, FRED M. (avec la collaboration de Donald W. OLIVER) (1970). Clarifying Public Controversy: An Approach To Teaching Social Studies. Boston: Little, Brown.

NEWMANN, Fred N. (1975). Education For Citizen Action: Challenge For Secondary Curriculum. Berkeley: McCutchan.

OlIVER, Donald W. et James P. SHAVER (1966). Teaching Public Issues in the High School. Boston: Houghton Mifflin.

RATHS, Louis E., Merrill HARMIN et Sidney B. SIMON (1978). Values And Teaching: Working With Values in the Classroom, $2^{\mathrm{e}}$ édition. Columbus, $\mathrm{OH}$ : Merrill.

ROKEACH, Milton (1969). Beliefs, Attitudes and Values. San Francisco: Jossey-Bass, p. 124.

SHAFTEL, Fannie R. et George Shaftel (1982). Role Playing in the Curriculum, 2e édition. Englewood Cliffs, NJ : Prentice-Hall, p. 157-159.

SHAVER, James P. et William STRONG (1976). Facing Value Decisions : Rationale-Building For Teachers. Belmont, CA: Wadsworth, p. 57. 